\title{
Footprints of the YORP effect in asteroid families
}

\author{
Paolo Paolicchi ${ }^{\mathrm{a}}$, Zoran Knežević ${ }^{\mathrm{b}}$ \\ ${ }^{a}$ Dept.Physics, University of Pisa, Largo Pontecorvo 3, I-56127 Pisa, Italy \\ ${ }^{b}$ Astronomical Observatory, Volgina 7, 11060 Belgrade 38, Serbia
}

\begin{abstract}
The YORP effect affects not only the rotation rate, but also the spin orientation of asteroids. For this reason, due to Yarkovsky effect, it influences also the evolution of the semimajor axis with time. In asteroid dynamical families, the combined outcome can be a depletion of objects in the central part of the the family, shown by the absolute magnitude vs. semimajor axis so-called V-plot. A reclassification of asteroid families, extended to a very large database of proper elements, has recently been performed by Milani et al. (2014). Only some of the related V-plots, used to estimate the age of several families, exhibit the depletion predicted above. In this paper we discuss the problem, introducing the concept of the YORP-eye and a general method of analysis. We show that the effect may sometimes be located in the low $H$ tail, and thus difficult to detect. Moreover, it may be hindered by several anomalous physical properties of the family (asymmetry, cratering origin, multiple collisions history and so on). With a new method of analysis, we identify the footprints of the effect for most of the analysed families, obtaining also an independent estimate of the age of the family. In spite of the uncertainties, we obtain a very good agreement between these ages and those estimated on the basis of the slope of the V-plot: a result which supports both methods and the underlying physics.
\end{abstract}

Keywords: Asteroids, Asteroids, rotation, YORP effect, dynamical families, age of families

Email address: paolo.paolicchi@unipi.it (Paolo Paolicchi) 


\section{Introduction}

The relevance of the YORP effect (Rubincam, 2000) for the observable properties of asteroids and, in particular, of asteroid families, has been repeatedly claimed in recent years. In particular the YORP effect affects the spin orientation, causing a general migration of the spin vector pole towards extreme obliquities measured from the normal to the orbital plane (Vokrouhlický and Čapek, 2002; Bottke et al., 2002, 2006; Micheli and Paolicchi, 2008; Nesvorný and Vokrouhlický, 2008; Vokrouhlický et al., 2015). Some footprints of this behaviour have been found in the general distribution of the spin vectors for bodies smaller than 30/40 km (Hanuš et al., 2011; Paolicchi and Kryszczyńska, 2012), while for families the clustering of the poles has been observed in the case of Koronis family (Slivan, 2002; Vokrouhlický et al., 2003). It is also assumed to be the cause of the increased number density of bodies close to the borders -in the semimajor axis- of several families (Vokrouhlický et al., 2006; Bottke et al., 2015), even if, as discussed by Spoto et al. (2015), this effect is not evident for all the families. The pole clustering pushes the objects towards the borders of families by enhancing the semimajor axis mobility due to diurnal Yarkovsky effect (Farinella et al., 1998; Farinella and Vokrouhlický, 1999; Bottke et al., 2002; Chesley et al., 2003; Vokrouhlický et al., 2015).

In this paper we analyse, with a method useful for statistical purposes, the properties of 31 families for which Spoto et al. (2015) estimated the age. We performed this analysis on the basis of the $a-H \mathrm{~V}$-shape plots, using a sample of more than 500000 objects (i.e. an extended sample also in comparison to that employed in the previous general paper on families by Milani et al. (2014), hereinafter referred to as paper I). The families have been classified according to the method defined in paper I.

\section{Great expectations: the YORP eye theory.}

In general, the YORP effect is assumed to cluster the spin poles close to $0 / 180^{\circ}$ of obliquity with respect to the normal to the orbital plane, on a timescale of the order of a YORP cycle (defined below); it is not exactly clear what happens later, but maybe the clustering is at least partially reversed, due to changes of the overall rotational properties (bursting fission, collisions). The duration of a cycle increases with the size of the asteroid, thus one may assume something of the following kind: for a given family 


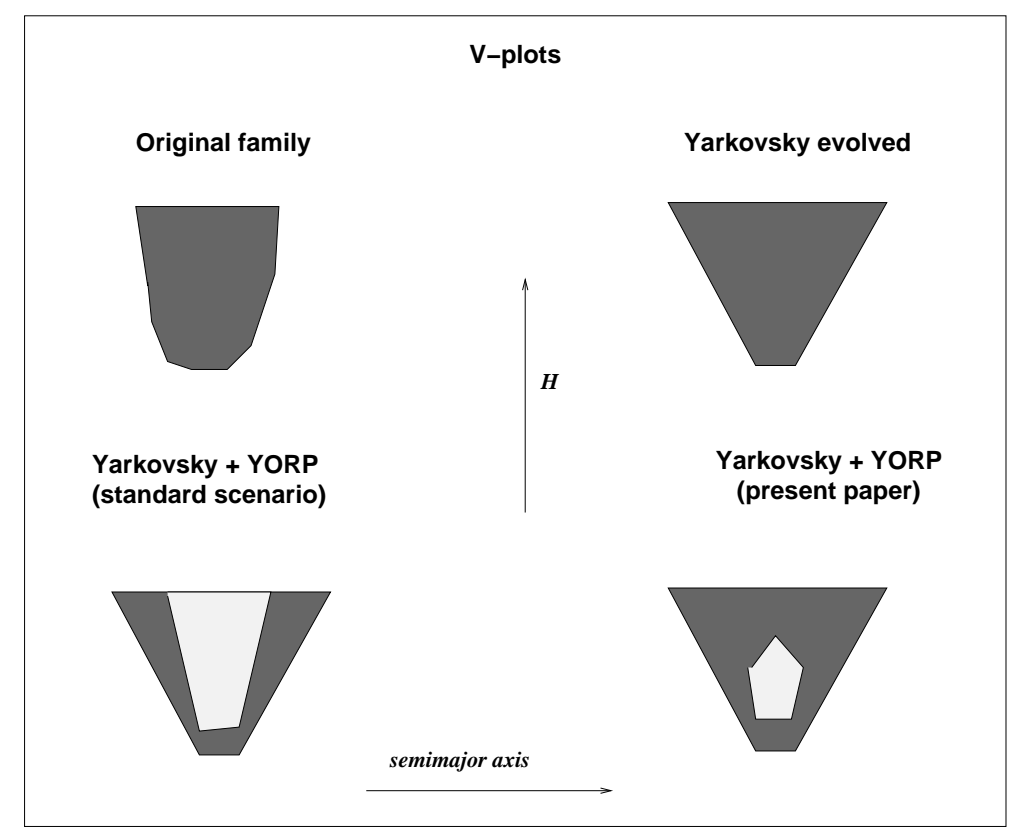

Figure 1: The figure schematically represents the expected appearance of the regions in the $a-H$ plane occupied by the members of a family ( $a$ is in abscissa, $H$ in ordinate). The top left figure refers to the original structure of the family, due to the individual properties of the colliding bodies, to the impact geometry, and so on: the shape is not clearly defined, even if a general trend towards a larger spread in $a$ for the smaller members can be expected. The top right figure corresponds to the shape of the family after some time, assuming only the Yarkovsky mobility: we get the classical "V-plot". Taking into account the YORP effect and clustering of the spin axes, we expect a depletion of bodies in the central regions of the plot, not affecting only the few largest members (bottom left plot). According to the ideas presented in this paper, however, the depletion may be present (or reach its maximum effectivity) in a limited range of $H$ (bottom right plot).

age, objects larger than a given value have not had time to be strongly affected by YORP; within a certain range of sizes the age of the family and the timescale of the cycle are similar, thus the clustering is very effective; for smaller objects several cycles have passed, thus it is not easy to assess the resulting distribution of the poles. Since the pole orientation directly affects the strength of the Yarkovsky effect, we can expect that at a given size range we should have a "void" in the $a-H$ V-shape plot; we are calling it the YORP eye. A schematic representation of the $\mathrm{V}$-plot according to various assumptions is given in Fig. 1. In the following we discuss the main idea of the YORP-eye theory. 
The variation in time of the rotational properties, obliquity $\varepsilon$ and rotation rate $\omega$, due to YORP effect, depends on the size $r$, albedo $A$, semimajor axis $a$ and momentum of inertia $I$ according to an equation of the form:

$$
d \varepsilon / d t=\operatorname{const}\left(f(A) r^{3}\right) /\left(a^{2} I\right)=\operatorname{constf}(A) /\left(a^{2} r^{2}\right)
$$

(the equation for $\omega$ is essentially the same apart from the different value and dimensions of the constant). $f(A)$ is a function of albedo, depending on the properties of the body (see below).

A typical timescale for a YORP cycle is defined by a relative variation of the rotation rate of the order unity or (which is more relevant for the present analysis) by an obliquity change of the order of a radiant:

$$
\tau_{Y-\text { cycle }}=\operatorname{const} \frac{a^{2} A r^{2}}{A f(A)}
$$

We have multiplied and divided by $A$ the right hand side term of 2 in order to introduce, in the next step, the absolute magnitude $H$, which depends on $A r^{2}\left(10^{0.4 H} \propto 1 /\left(A r^{2}\right)\right)$.

Let us consider two bodies: one with properties $(H, a, A)$, and the other, reference one, $\left(H_{0}, a_{0}, A_{0}\right)$. By writing down Eq. (2) for both of them, and dividing the obtained expressions, we straightforwardly get the relation between the YORP cycles of the two bodies:

$$
\tau_{Y-c y c l e}(H, a, A)=\tau_{Y-c y c l e}\left(H_{0}, a_{0}, A_{0}\right) \frac{a^{2} A_{0} f\left(A_{0}\right) 10^{0.4 H_{0}}}{a_{0}^{2} A f(A) 10^{0.4 H}}
$$

We can now define the YORP age of a family such that for the same YORP age members with magnitude $H$ equal to some reference, arbitrarily chosen, magnitude $H_{0}$, undergo a similar phase of their YORP-driven evolution. $Y_{\text {age }}$ should be equal or proportional (through the inverse of the constant defined in Eq. (2)) to the number of YORP-cycles which a family member with $H=H_{0}$, but with semimajor axis $a$ and albedo $A$, has undergone during its lifetime (assumed equal to the age $\tau_{f}$ of the family). Thus we have:

$$
Y_{\text {age }}=\frac{\tau_{f}}{\tau_{Y-c y c l e}\left(H_{0}, a, A\right)}=\frac{\tau_{f} A f(A) a^{-2}}{\tau_{Y-c y c l e}\left(H_{0}, a_{0}, A_{0}\right) A_{0} f\left(A_{0}\right) a_{0}^{-2}}
$$

where also $a_{0}$ and $A_{0}$ pertain to the arbitrarily chosen reference object. 
Note that the value of $Y_{\text {age }}$ in the first of Eq. (4) depends on the choice of $H_{0}$ :

$$
Y_{\text {age }} \propto 10^{0.4 H_{0}}
$$

There is, obviously, a degree of freedom in the definition, thus the value of $Y_{\text {age }}$ can be multiplied by an arbitrary factor, not necessarily dimensionless; in this way we can also get rid of the dimensions, and consider $Y_{a g e}$ as a dimensionless parameter; the only requirement, for our purposes, is that this "normalizing" factor is always the same across the various families.

Thus, to facilitate the computation, we can simply define a dimensionless $Y_{\text {age }}$ with the equation obtained from the second Eq. (4):

$$
Y_{\text {age }}=\tau_{f} A f(A) a^{-2}
$$

where $\tau_{f}$ is in $M y$ and $a$ is in $a u$.

The basic model of the YORP effect, according to Rubincam (2000), takes into account both the reflected and the re-radiated radiation, thus we can approximately assume $f(A)=1$ (see the discussion in Sect. 3). The $Y_{\text {age }}$ defined by Eq. (6) is perfectly fit to compare the properties of families for what concerns the YORP effect, but it is also useful to introduce a calibration, both to estimate the region of $H$ in which we expect to find the "eye" or something of the kind, and also to obtain, from its location, an indication of the age of the family, to be compared with other independent age estimates (see Sections 5 and 6). However, due to the various uncertainties which we will discuss in Sect. 3, and to the limited direct observations of the effect available (Vokrouhlický et al., 2015), only a rough calibration (not much better than on an order-of-magnitude level) is possible. We have, for this purpose, taken into account some estimates of the YORP cycle timescale concerning asteroid (951) Gaspra (Rubincam, 2000; Čapek and Vokrouhlický, 2004; Bottke et al., 2006).

According to Rubincam (2000), Fig.7, the $\varepsilon$ YORP cycle timescale of Gaspra is of the order of $1 \mathrm{~Gy}$; we recall that the semimajor axis and albedo for Gaspra are $a=2.2$ au and $A=0.22$. Thus, according to Eq. (6), a YORP age of about $45 \simeq 10^{3} \cdot 0.22 /\left(2.2^{2}\right)$ implies that a body of Gaspra's absolute magnitude $(H=11.5 \mathrm{mag})$, albedo and location, and age $\simeq 1 G y$, has undergone a single full YORP cycle, causing its obliquity to become close to $0 / 180^{\circ}$. The required time can be smaller if the original obliquity 
is far from $90^{\circ}$. This duration of a YORP cycle for Gaspra is also order-ofmagnitude consistent with the few data coming from the direct observations (Vokrouhlický et al., 2015) (we recall that the YORP timescale depends on $r^{2}$ ). Thus, even if the above value for Gaspra, obtained with a simplified model, may be overestimated by a factor ranging from 2 to 4 (Rubincam, 2000; Capek and Vokrouhlický, 2004; Bottke et al., 2006) we have taken it to fix our calibration. We can thus estimate the $H$ value for which a body with a given YORP age has undergone one cycle during its lifetime as:

$$
H_{1 \text { cycle }} \simeq 15.6-2.5 \log _{10} Y_{\text {age }}
$$

Thus, footprints of YORP evolution should show up approximately at $H=18$ for $Y_{\text {age }}=0.1, H=15.6$ for $Y_{\text {age }}=1, H=13.1$ for $Y_{\text {age }}=10$ and $H=10.6$ for $Y_{\text {age }}=100$. Note that one could also use different normalization for Eq. (7): assuming $Y_{\text {age }}=1$ for Gaspra, Eq. (7) would remain the same, except for the constant on its right hand side which would amount to 11.5.

Apart from the uncertainties, which we will discuss in Section 3, some offset from these values can be expected. In fact, after a YORP cycle, the spin axes are aligned, but the accelerated Yarkovsky mobility has not commenced immediately, that is from the cycle's beginning; thus the creation of the "eye" might be delayed. In principle, since the Yarkovsky effect depends on the size less steeply than the YORP one, this delay should be comparatively more significant for the smallest objects.

The width of the YORP-eye region is not easy to define, but should be at least on the order of a factor two in the timescale, that is corresponding to something like 0.75 mag.

\section{A realistic view}

Unfortunately, the analysis presented in the previous Section is simplified and based on very optimistic assumptions. In the reality several major sources of error or uncertainties play a major role, and we should not be surprised not to see a very strong effect in all the families.

The main sources of error are:

- Family membership: The presence of interlopers is a well known problem. Moreover, there is a possible bias introduced by the classification method (the multi-step method defined in paper I, tuned in such a 
way to avoid the chaining effect, is rather conservative in the membership assessment, especially for small objects); since the peripheral regions are populated by small objects, this may additionally enhance the "limb effect" (see below).

- Asymmetry of the family: The initial distribution of the family members in the space of orbital elements can be strongly asymmetric. We recall that, as stated in paper I, several families arise from cratering impacts, which are intrinsically asymmetrical; also the catastrophic fragmentation events create an asymmetric cloud of ejecta; moreover one has to add the effects of the usual oblique impact and of the overall angular momentum of the colliding system. This original asymmetry can be strong also along the semimajor axis. Finally, dynamical effects, in particular mean motion resonances, can produce asymmetries in the long term, or even terminate some families in semimajor axis.

- Family "limb-effect": This notion refers to the concentration of the members of a family in the central parts of the $\mathrm{V}$-plot, as a consequence of the family formation process. Let us consider, for instance, a family just formed with fragments ejected with a perfect spherical symmetry, and with a distribution of ejection velocities in a given range $|V| \leq V_{\max }$, for which every velocity vector in this range has the same probability. Also in this simplistic case the initial V-plot should not be uniformly populated, and the number frequency distribution of semimajor axes of family members would present a central peak (such as in Fig. 2). This initial clustering can affect the values of the central depletion parameter defined in Section 4).

- Multiple collisional events: Several families have been affected by multiple collisions; in some cases these different events cause also an asymmetry in the V-plot (see, for instance, the case of Vesta family in Paper I), and different age estimates come out. Even in the case that the overall structure of the family is dominated by one event, secondary collisions, which involve not only the largest remnant but also the other family members, can cause a random walk in the orbital elements space (Dell'Oro and Cellino , 2007), introducing a noise in their distribution. They can also affect the spin orientation.

- Other peculiarities in the original properties: The initial spin vector 
distribution may be unusual, correlations between the spin vector and the original semimajor axis can be present (La Spina et al., 2005; Milani et al., 2014). The timescale required for the final orientation of the spin, due to YORP, may depend also on the original spin properties; it may introduce a further noise in the data. Moreover the evolution can be affected by various dynamical effects due to resonances, spin orbit interactions, and so on.

- Albedo and thermal conductivity: We usually know the geometrical albedo (albeit often with large uncertainties), which is used also in the definition of $H$. The influence of the albedo on the YORP effect (the term $f(A)$ introduced in the previous Section) is a very complex problem. In the zero-thermal-conductivity approximation, used in the original studies on the YORP effect (Rubincam, 2000), assuming also an isotropic reflection and reemission of the light, the albedo is not relevant. In reality, the thermal conductivity is non-zero and the scattering properties of the light are not consistent with an ideal Lambertian behaviour. Thus the situation is not so simple, some dependence on the albedo may be present, but the relevant albedo is neither the geometric one, nor exactly the Bond albedo, but something different (Breiter and Vokrouhlický, 2011). Note that in the limit of a very large thermal conductivity only the reflected light should be important, and thus $f(A) \simeq A$, but the case applies only to very small, and thus unobservable, bodies. Our assumption $f(A)=1$ is an approximation, but the introduced uncertainties do not seem to be critical.

- Uncertainties in the $H$ estimation: The estimated value of $H$ may be affected both by significant uncertainties and by some systematic bias (Pravec et al., 2012; Vereš et al., 2015). The uncertainties result in an erroneous position of a body on the $\mathrm{V}$-plot, thus essentially representing a noise term. Possible systematic effects may introduce errors difficult to estimate. Moreover $\mathrm{H}$ is constant only for spherical objects, but it changes from an apparition to the other, being a function of the aspect angle, for non-spherical objects. This complicated effect introduces some further noise which is in addition to the usual uncertainties due to poor photometric measurements and extrapolations of few data to zero phase angle. Due to these problems an upgraded photometric system for asteroids has been introduced (Muinonen et al., 2010). 
- Behaviour of YORP effect with bodies of different shapes and properties: The "typical" YORP effect holds only for some "typical" shapes (essentially with few major irregularities). Bodies of a more complex shape may have a different (and, usually) a weaker and slower YORP evolution (Micheli and Paolicchi, 2008; Statler, 2009). The uncertainties cause a strong scatter of the individual evolutionary timescales: also the overall calibration of the effect, i.e. the estimate of an average value, which is of paramount importance in a work like the present one, can be affected by a significant uncertainty. Note that, as discussed in the previous Section, we have very few observational estimates of the effect, which might suffer, in principle, unknown biases; combining this scarcity of data with the difficulties in modelling we can easily estimate a relative uncertainty of at least a factor two.

- Progressive readjustments of the asteroid shapes and influences on YORP cycles: The influence of YORP effect on the spin rate and also on the spin axis orientation may be changed even by moderate adjustments of the shape, due, for instance, to non-catastrophic collisions (Cotto Figueroa et al., 2015; Bottke et al., 2015).

- Long term evolution of YORP effect. While the behaviour of bodies during the first YORP "cycle" is fairly well understood (even with the uncertainties discussed above), the subsequent evolution is very uncertain.

- The "YORP-eye" may corresponds to values of $H$ for which there are not enough bodies: it is a typical problem for families with a very small or a very large YORP-age (very young families, preferentially with dark members, or very old families, especially with moderately high reflectivity). In the former case observational selection effects hinder the discovery of a sufficient number of very small objects; in the latter the interesting region involves rather large objects, which are naturally few.

All these uncertainties act together; thus it is not obvious that the general features, identified in the previous Section as consequences of the YORP effect, may be really observable. 


\section{The central depletion parameter}

The previous considerations, explaining, as pointed out by Spoto et al. (2015), why the expected central depletion due to YORP effect is not always immediately noticeable, led us to attempt a statistical analysis of the available sample, with the goal of identifying not only the most obvious footprints, but also the most degraded ones. Thus we are not looking for sharply defined voids (or "eyes") in the $a-H$ plots for various families, but we search for the dependence on $H$ of some general central depletion parameter. In doing this, we will follow the approach already discussed in the literature (Pravec et al., 2002; La Spina et al., 2004; Kryszczyńska et al., 2007; Paolicchi and Kryszczyńska, 2012).

To define a central depletion parameter, we have divided the $a$-range of the family, for a given range of $H$, into seven bins, corresponding to equally spaced intervals in the sine of the angle $\varepsilon-\pi / 2$. Namely, if we assume that the value of the semimajor axis $a$ depends mainly on the Yarkovsky effect, the change in $a$ due to the evolution, and thus the present value, depends on the sine of this angle. Thus, if the obliquity angles are randomly oriented, we should expect a uniform distribution of bodies in the seven bins. The division in terms of the sine can be justified by a simple probabilistic consideration: if the spin axes are randomly oriented, the probability of having a spin with a given obliquity $\varepsilon$ scales with the circumference of the parallel, on the unit radius sphere, at the same latitude, i.e. with $\cos (\varepsilon-\pi / 2)$. Thus the probability of having an inclination between two values of $\varepsilon$ is computed by integrating over $\varepsilon$, and is hence proportional to the difference between the sines of the interval boundary angles.

Since YORP effect acts in favour of increasing the population close to the borders, we have defined a central depletion parameter:

$$
R=\frac{3 N_{\text {ext }}}{4 N_{\text {int }}}
$$

where $N_{\text {ext }}$ is the summed population of the four lateral bins and $N_{\text {int }}$ that of the three central ones. Thus, $R$ should be equal to one for isotropy, and should be larger than one in the case of YORP-induced border clustering. However, the matters can be completely different if other effects are at work, such as the above mentioned "limb effect". In such a situation, $R$ can be significantly less than one, in spite of the depletion induced by YORP effect during the evolution. An example of a family for which this latter effect 
seems to be dominating is given by Fig. 2 .

Note that the central depletion parameter is actually related to the usual definition of kurtosis in statistics (the fourth moment scaled by standard deviation); nevertheless, we decided to employ a more intuitive definition. A systematic analysis of the momenta of various orders might, however, be useful, taking into account for instance the third moment - skewness (essentially the asymmetry), and also momenta of higher order. A more formal analysis could be connected to a general evolutionary model of the semimajor axis distribution, which we are intending to develop in the future.

Let us now analyse the $R<1$ case in more detail by using the simplistic spherically symmetric ejection case described in the previous Section. At least if a low-eccentricity approximation holds, the drift in the semimajor axis $\Delta a \propto \Delta V_{T}$, where $V_{T}$ is the transversal component of the ejection velocity. Hence, $\sin (\psi)=V_{T} /\left|V_{\max }\right|$, and thus also $\Delta a / \Delta a_{\max }=\sin (\psi)$.

If we now consider a sphere that envelopes the velocity vectors translated to the common origin, and define its "equator" as the plane that corresponds to $\psi=0$, the intersection of the plane parallel to this equator, corresponding to an arbitrary angle $\psi$, and the sphere, is a circle of radius $V_{\max } \cos (\psi)$, whose area is thus proportional to $\cos ^{2}(\psi)$. To find the plane which splits the volume of one hemisphere in two equal parts, we need to determine the median of $\psi$, given by the equation:

$$
\int_{\gamma}^{\pi / 2}(1+\cos 2 \psi) d \psi=\int_{0}^{\gamma}(1+\cos 2 \psi) d \psi
$$

resulting into a $\gamma$ value of the order of $10^{\circ}$. Obviously, this median angle also halves the set of transversal velocities, and thus of the semimajor axis drifts. In conclusion, $\gamma$ being such a small value means that we would have a large concentration of objects in the central bins of our division used to compute the central depletion parameter. As a matter of fact, in the considered simplistic case, $R$ should be of the order of 0.5 .

Note that this low value could actually be even lower: the above simplistic model that we considered corresponds to a distribution of $|V|: d n(|V|) \propto$ $|V|^{2} d|V|$, which could be biased in favour of the peripheral regions, thus a more refined model should further reduce the central depletion.

What happens at a later time? The Yarkovsky effect will spread the bodies according to the obliquity of their spin axis, and a V-plot, now completely dominated by the Yarkovsky-driven evolution, should again have a 


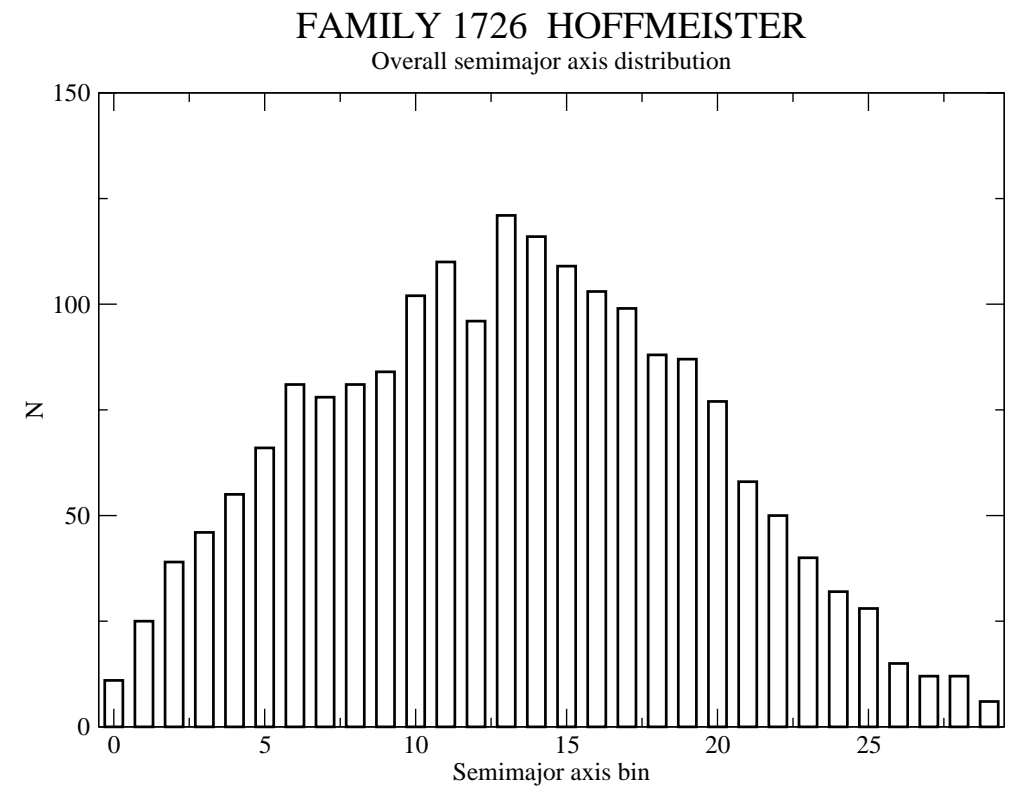

Figure 2: The number frequency distribution of the semimajor axis of the family 1726 , within the range of the same, divided into thirty equal bins. The distribution is evidently peaked in the central region, exhibiting the dominance of the limb effect.

central depletion parameter close to 1 . However, the situation may be more complex: the Yarkovsky effect and the original properties are combined in reality, and the latter are usually important -and sometimes dominant- in the region of larger bodies (low $H$ ). Moreover ejection velocities and original spin axis may be correlated (see the discussion in Paper I), and thus the situation might again be different. We are planning to analyze this point in more detail in the future. For now, we can claim that the cases of "low central depletion maximum", which we will discuss later, are well understood and not really troublesome.

In some cases the analysis may be complicated by the strong asymmetry of the family itself. An example is given by the family 847, for which the overall histogram of the semimajor axis is dominated by the strong right-left asymmetry (Figure 3, top panel). The representation of the V-plot of the same family (bottom panel) clarifies the problem. According to Spoto et al. (2015) the family has a complex structure (presumably with contamination by another family, namely 3395); family of (847) Agnia centers at about $2.78 \mathrm{au}$, and that of (3395) Jitka centers at $2.79 \mathrm{au}$. The slopes of the V-plot 
lead to two rather different age estimates, even if, in this case, one is by far more reliable than the other (Spoto et al., 2015).

Note that in this case one might conspectu identify a weak footprint of the YORP clustering in the middle of the right wing, at $H$ values around $14-16$, while the automated procedure described below finds the required footprint, but at lower values of $H(12-13)$, where the density of members is by far lower.

In the present paper we have analyzed the families listed in Table 1 . In Spoto et al. (2015) the ages are computed by using the slopes of the V-plot. The two slopes, and thus the ages, are usually different. However, in many cases the ages are very similar, indicating the existence of a single collisional family; in such cases, we have taken the average of the two values. In other cases the two ages differ significantly, a possible indication of a twofold (or multiple) collisional process. Since the purpose of the present paper is a statistical assessment of the YORP effect and a statistical comparison between the ages computed by the $\mathrm{V}$-plot slopes and those estimated according to our method based on YORP effect, we did not enter into the detailed analysis of individual families. In most cases we have simply "duplicated" the computations for the family with two possible ages. However, when, as in the case of the family 847 (see above), according to Spoto et al. (2015) the age computed by the inner $\mathrm{V}$-plot slope is probably the correct one, we have retained only this value.

Obviously, we also list the YORP ages, computed according to Eq. (6).

For every family we have computed a running value of $R$ as a function of $H$. The size of the running box is 100 objects. Moreover, to facilitate the reading of the figures, we have grouped the various points within a $H$ range of 0.5. Thus the effective boxes are more populated whenever we have many bodies in a short $H$-range, a typical case in the small sizes region for the largest families. The use of rather rough $R$ estimates may seem overrestrained, since we have at our disposal a lot of data, and one could perform a running-box analysis in $a$ for every region, looking at density minima, and so on. However, as discussed above, the real families are affected by several noise effects, thus the more refined analysis (which we have tried) often leads to confusing results. Even this less ambitious choice of analyzing the population in the seven bins as function of $H$ is not always reliable and completely fruitful. For instance, we represent here the case of the family 163, completed with the addition of family 5026 (this choice is supported by previous evidence, see for instance Spoto et al. (2015)). The family is a 
FAMILY 847 AGNIA (+3395 JITKA)
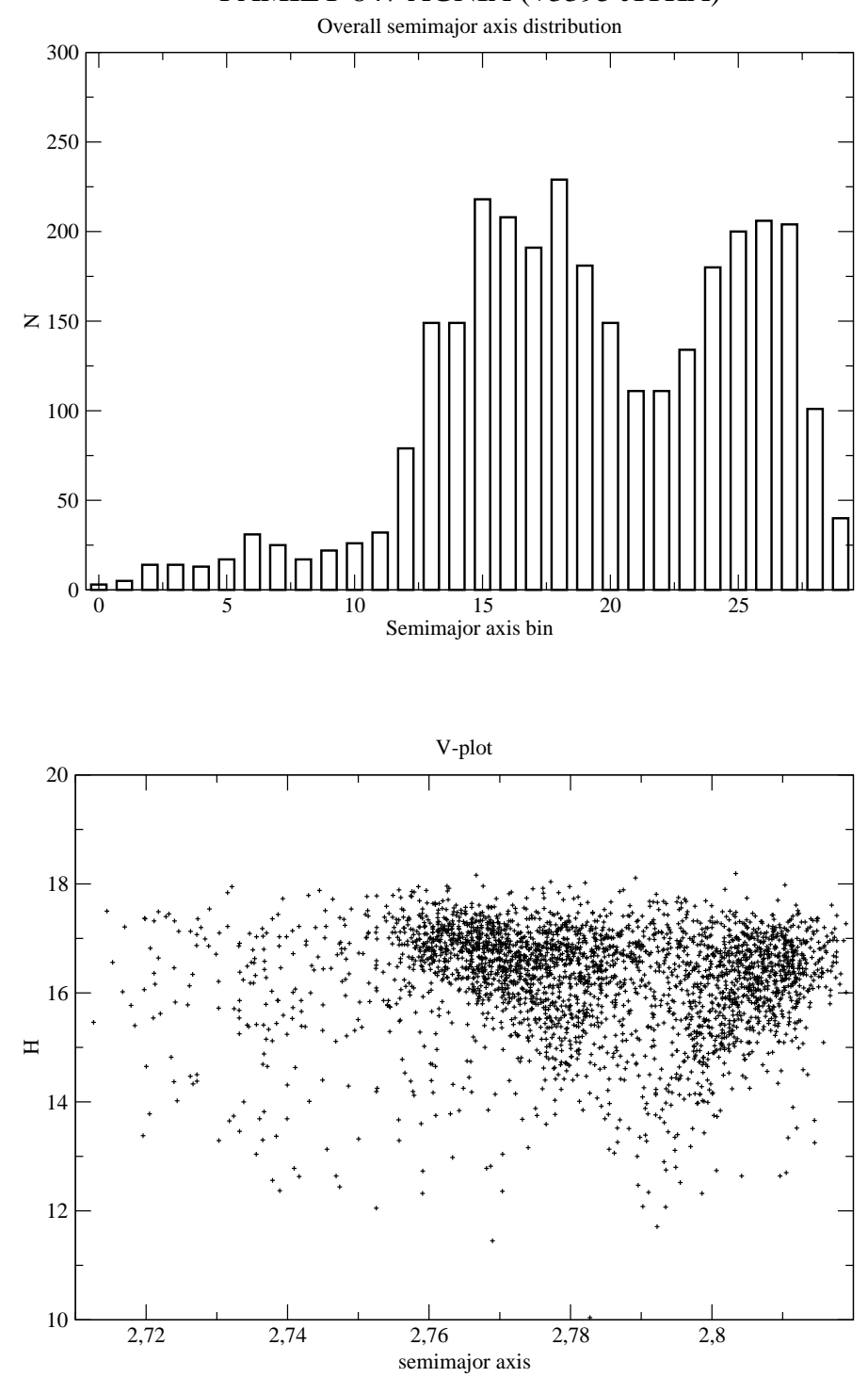

Figure 3: Top panel: The number frequency distribution of the semimajor axis of the family $847+3395$, within the range of the same, divided into thirty equal bins. The distribution is evidently asymmetric, due to the overlapping with family 3395. Bottom panel: The V-plot of the family ( $H$ vs $a$ ) exhibits a strong clustering for $a>2.76$ au due to family 3395. In the middle of the clustering one can find a potential YORP-eye; the gap which extends towards lower $H$ is centered with respect the members of family 847 . 
FAMILY 163 ERIGONE (+5026 MARTES)



Figure 4: The V-plot of the family $163(H$ vs $a)$. The plot exhibits a marked gap in the central region.

spectacular example of the central gap due to YORP effect, as shown by the Figure 4; however, if we plot the behaviour of the population in the seven bins, as function of $H$, we obtain a rather confusing outcome: the bin 4 (the central one) is poorly populated, but the same is true also for bin 7 (one of the extremes) and, in a wide range of $H$, also for the other extreme bin 1. Thus the central depletion effect is essentially driven by the lateral bins 2 and 6 , corresponding, in the sense discussed above, to moderate spin axis inclinations $\left(25^{\circ}-45^{\circ}\right)$. The result are represented in the two panels of Figure 5. Obviously this complex structure is due to various causes. The joined family occupies a range in semimajor axis such that it ends up in resonances 10/3 with Jupiter (at $2.33 \mathrm{au}$ ) and 2:1 with Mars (at about $2.41 \mathrm{au}$ ). It is also of cratering type, that implies mostly small members. Objects above $2.4 \mathrm{au}$ are affected by the resonance. In the $(\mathrm{a}, \mathrm{e})$ projection of the family the objects in the high semimajor axis tail form narrow "jet(s)", which could be due to the HCM chaining effect. In conclusion, the "lack" of objects in bin 7 could probably be due to the combination of all these causes, but it is not possible to establish this firmly. 
FAMILY 163 ERIGONE (+5026 MARTES)
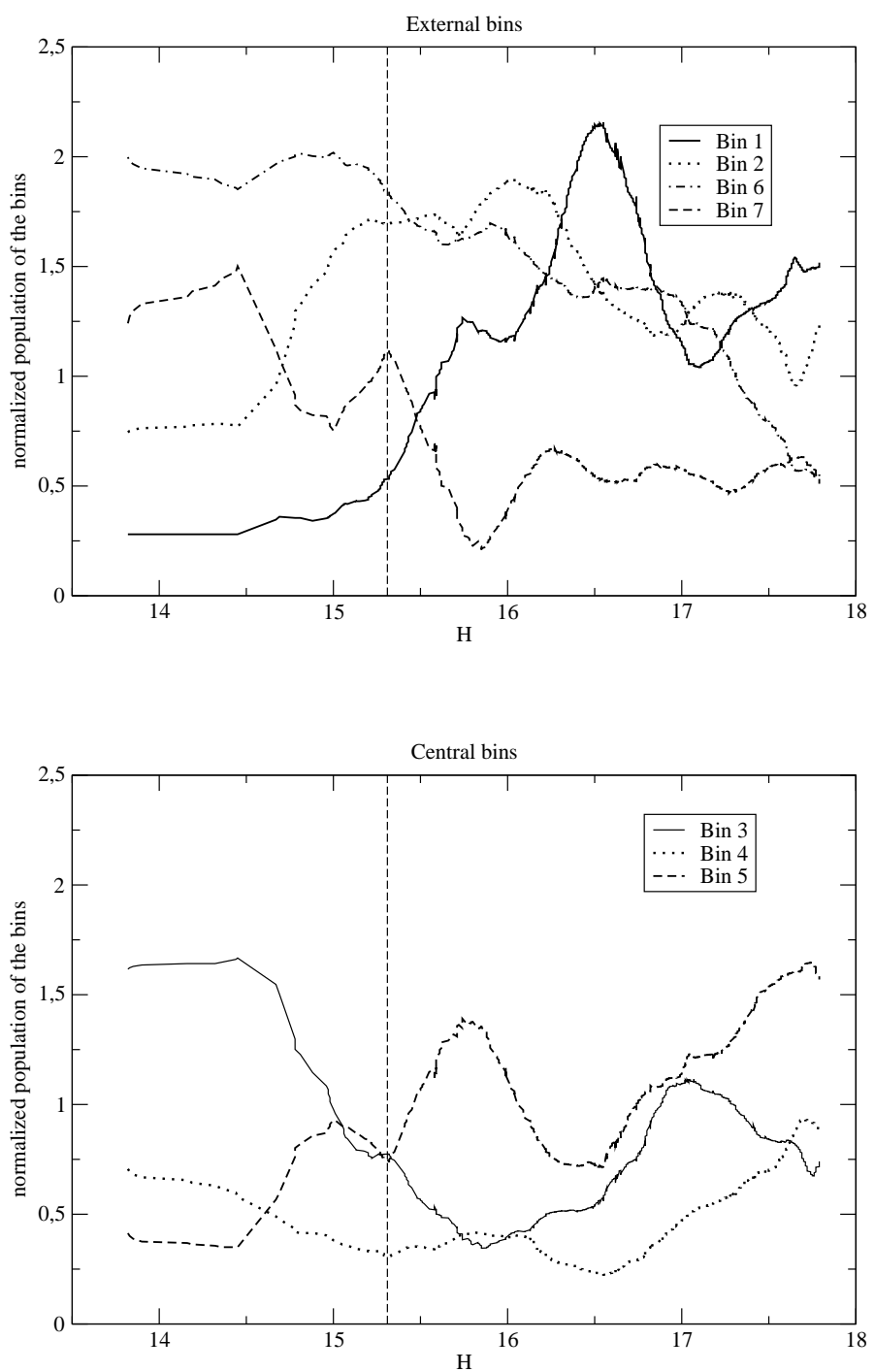

Figure 5: The population of the seven bins, introduced in the text, corresponding to various ranges in semimajor axis (and thus, in principle, of obliquity) as functions of $H$, for family $163+5026$. The top panel represents the four external bins (the population is normalized to the average value within the bins); the vertical dashed line corresponds to the chosen $\mathrm{R}$ maximum (see text). The bottom panel represents the three central bins. 


\section{Results and discussion}

As discussed in the previous Section, the computations have been performed to analyze the dependence of the central depletion parameter $R$ on the absolute magnitude $H$, starting from the population of the seven bins, as represented in Fig.5. We have also introduced a smoothing procedure described in Sect. 4 to avoid short scale fluctuations, which are, obviously, of no interest. We compare the results with the expected $H$ value for which the maximum of $\mathrm{R}$ should occur, assuming that the estimate of the age, given by Spoto et al. (2015) and used as discussed in Section 4, is perfectly correct. However, we opted for simplifying the graphical representation of the results in the Figures by representing, instead of the entire $H-R$ curve, only the points of the same curve corresponding to local maxima. The figures are easier to read, while no significant information is lost, neither for what concerns the shape of the curve nor - essentially - for what concerns the relevant range. Note that in the same plot one can also represent the location of the maximum expected according to the estimated age of the family. As an example, we represent both - curve and maxima - for the family 3, in Figure 6. The family has been chosen since the expected maximum is out of the range of the data (see later in the text for the discussion). This property and the related conclusions are not affected, in this and in all the other cases, by the chosen representation.

We have performed the computations described above for the 31 families listed in Table 1. For instance, in Fig. 9 we represent (top panel) the local maxima we have found in the case of family 163, and compare them with the expected location of the maximum. The same has been done for all the families in our sample (see the discussion regarding Fig. 6, and also Figs. 7, 10, 11, 13 and 14). We choose, from these plots, the significant maximum (see below), for which we have a more relevant depletion. The "computed" maximum indicates the $H$ value for which one can find the YORP eye in the V-plot.

The synthesis of the results is given in Table 2 .

As already mentioned, the various families behave differently with respect to our analysis. The main "variable" is the expected $H$-value for the fulfillment of a single YORP cycle, i.e. the potential center of the "YORP-eye". The resulting values range between 10 and 17 . We note that most of the family members are rather faint, with $H>15$ mag. Thus in several cases the most interesting region is poorly populated, or not populated at all. In 
FAMILY 3 JUNO

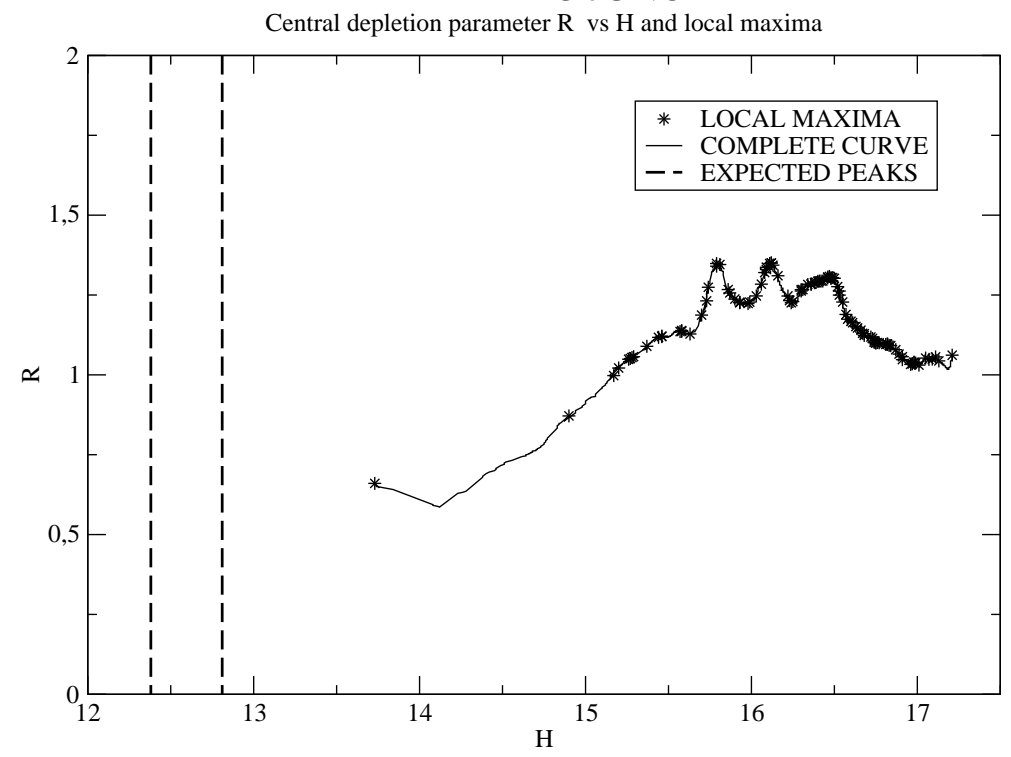

Figure 6: The $H-R$ curve for the family 3 , with superimposed points corresponding to the local maxima. We recall that larger values of $R$ correspond to a more effective depletion of bodies in the central bins. It can be seen that the maxima fairly well represent both the overall behaviour of the curve and the significant range. The dashed vertical lines represent the expected maxima, according to the two age estimates. 
FAMILY 3815 KONIG

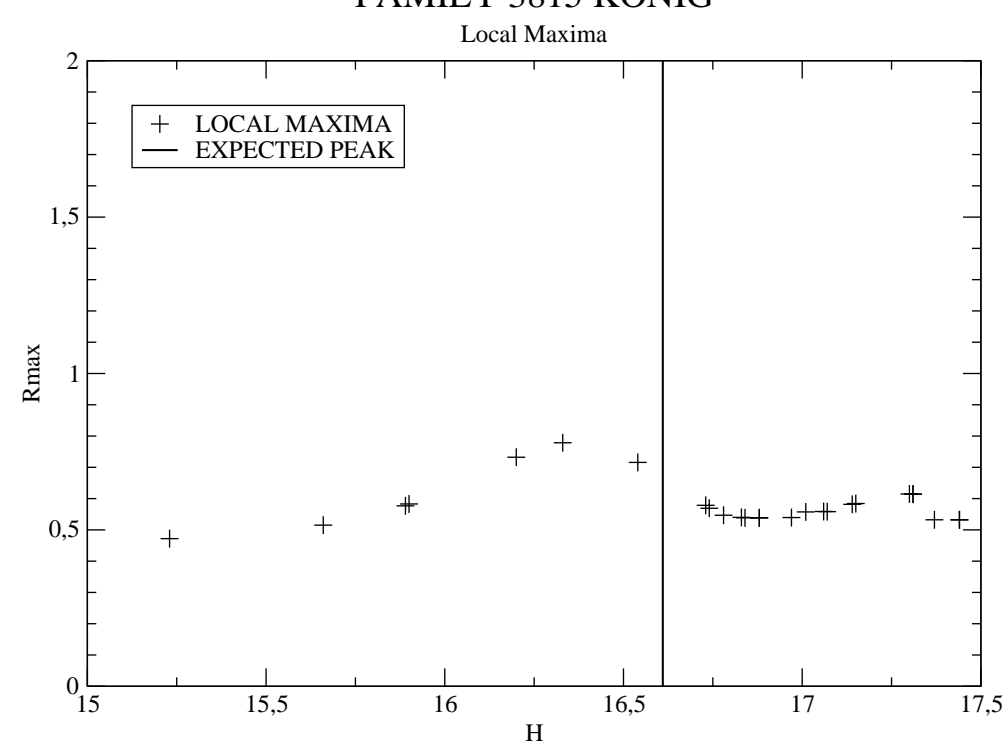

Figure 7: The maxima of $R$ as function of $H$, compared to the expected $H$ for the maximum, for family 3815 , obtained by using the nominal age and other relevant parameters.

three cases the expected maximum is completely out of the range of our data, as shown for the family 3 in Figure 6, and they have been labelled as "OUT".

Note that these three cases remain critical also after the re-calibration of the YORP-timescale, discussed below. In effect, after the recalibration, the expected peaks are shifted closer to the local maximum corresponding to the lowest $H$-value; however these maxima correspond to very low values of $R$.

The analysis of the remaining 28 families has led to some interesting results, but, as one could expect, not all the results are equally satisfactory.

First of all, the maximum value of $R$ may be rather low. It is not a surprise: we have shown that the sole "limb-effect" can enhance the population in the central bin by a factor two. In this regard, the (five) cases for which $R<1$ (but always larger than 0.5) are acceptable in principle. However, in all these cases the semimajor axis distribution for $H$-values close to the maximum does not exhibit any strong gap. We illustrate this situation with the example of family 3815, in Figures 7, 8 (top and bottom panel). Looking at the latter two figures, one cannot find the expected gap, neither in the histogram, nor in the V-plot, even if the central regions are slightly less populated around the computed maximum. 
FAMILY 3815 KONIG
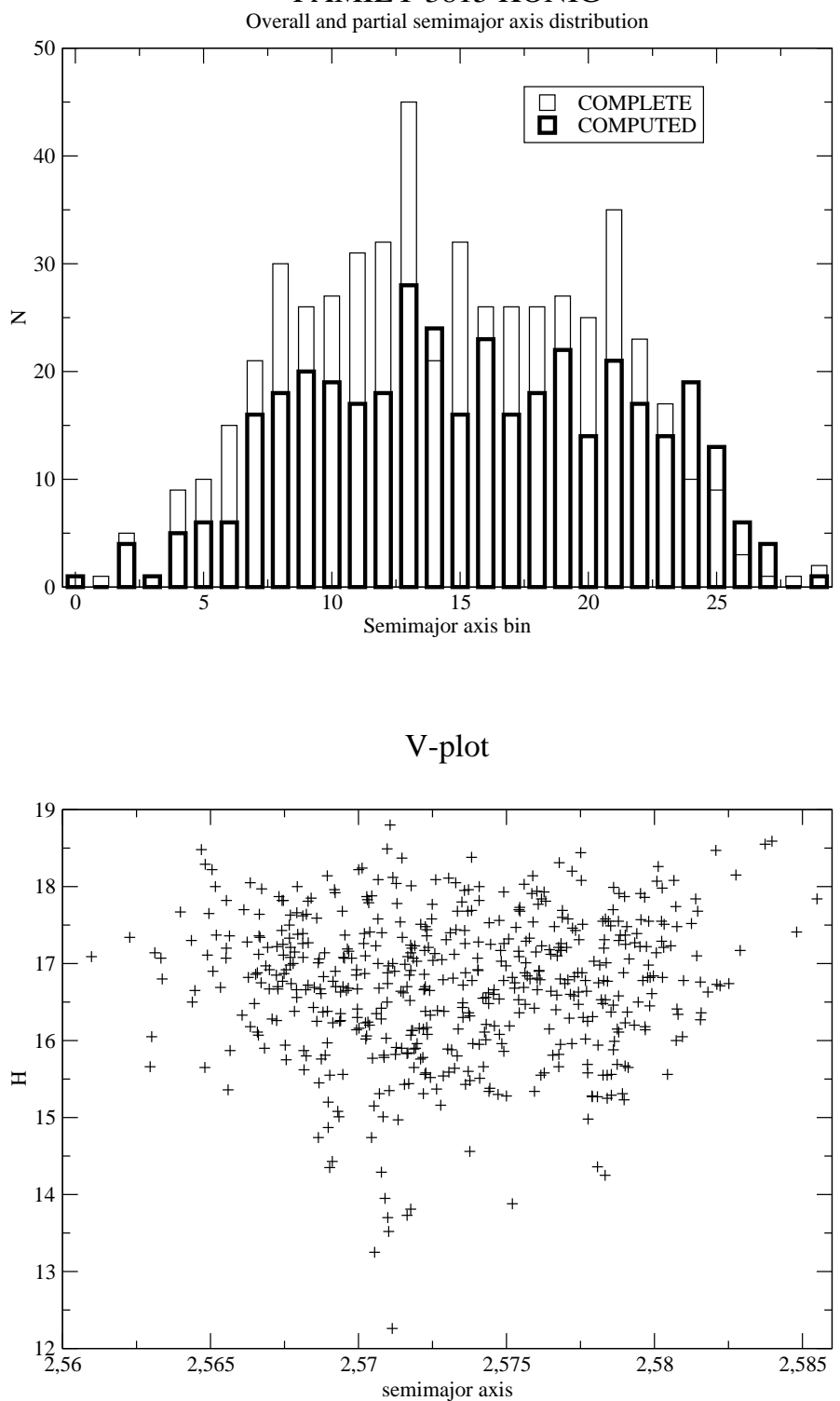

Figure 8: Top panel: The distribution of asteroids in 30 bins by semimajor axis, for family 3815 . We represent the complete family and the subsample of bodies with $H+/-$ $1 \mathrm{mag}$ around the computed maximum of $R$ (referred to as "computed"). Here and in the following figures the partial histograms are shown to emphasize the enhanced central depletion close to the maximum. Note that the range in $a$ refers to the relevant sample, thus it is not the same for the two cases: the paradox of having, in some bins, more objects in the partial sample, is not real. Bottom panel: The $\mathrm{V}$ - plot of the family does not explicitly evidence the YORP-driven clustering. 
Another critical issue concerns the presence of several sharp maxima in the $H-R$ plot. This is again not surprising a-priori: the original properties of the family can give rise to maxima before the changes due to YORP take place. They may involve also large bodies, and thus stand unaffected by a subsequent YORP evolution on the left of the expected YORP-cycle maximum, or may originally be quite high: for instance, a family formed by cratering may exhibit a strong original central depletion. Moreover, we don't know much about the long term evolution, after several YORP-cycles; the possibility of further clustering, even with some periodicity, cannot be excluded, and the presence of sharp maxima on the right of the expected maximum, and thus involving faint objects, is possible. There is no a priori reason why these additional maxima should be lower than that due to the YORP-cycle.

Thus, when the absolute maximum is not very far from the expected location, we have taken it as the significant one. On the contrary, whenever the absolute maximum is too far and there is a closer recognisable secondary maximum, we have chosen the latter. Out of the 28 analyzed families, 11 exhibit such a problem. However, there are important differences among them. Some cases are only "formally" a problem. For instance, the already discussed family 163 presents a local maximum (with $R>2$ ) not far from the expected $H$, and a moderately higher absolute maximum at higher magnitudes (Figure 9, top panel). As it is easy to see from Figure 4, this second maximum is connected to an anomalous concentration of bodies in the left wing, at magnitudes around $16-17$. The reason is obscure (maybe a secondary event?). However, we can be sufficiently confident that the local maximum is significant, and use that one for our Table. In effect, this family presents something as a "YORP-valley", and the central depletion can be easily found for different subsamples (the central depletion is apparent in the histogram of the complete family, and is even more marked in the subsample related to the "computed" maximum, but is substantial also in the subsample around the "expected" maximum, see Figure 9, bottom panel).

In the two cases (families 15 and 24, labelled as (3) in Table 2) the adopted secondary maximum is almost at the same level as the absolute maximum, while for family 20 (labelled as (4)) the secondary maximum which we adopted, and which is above unity, is the leftmost one, but still to the right of the expected value. In the expected YORP-eye region there are very few (bright) bodies. The situation may be clarified by Figure 10.

In the remaining 7 cases (labelled as (5)), the secondary maximum, that 
FAMILY 163 ERIGONE (+5026 MARTES)
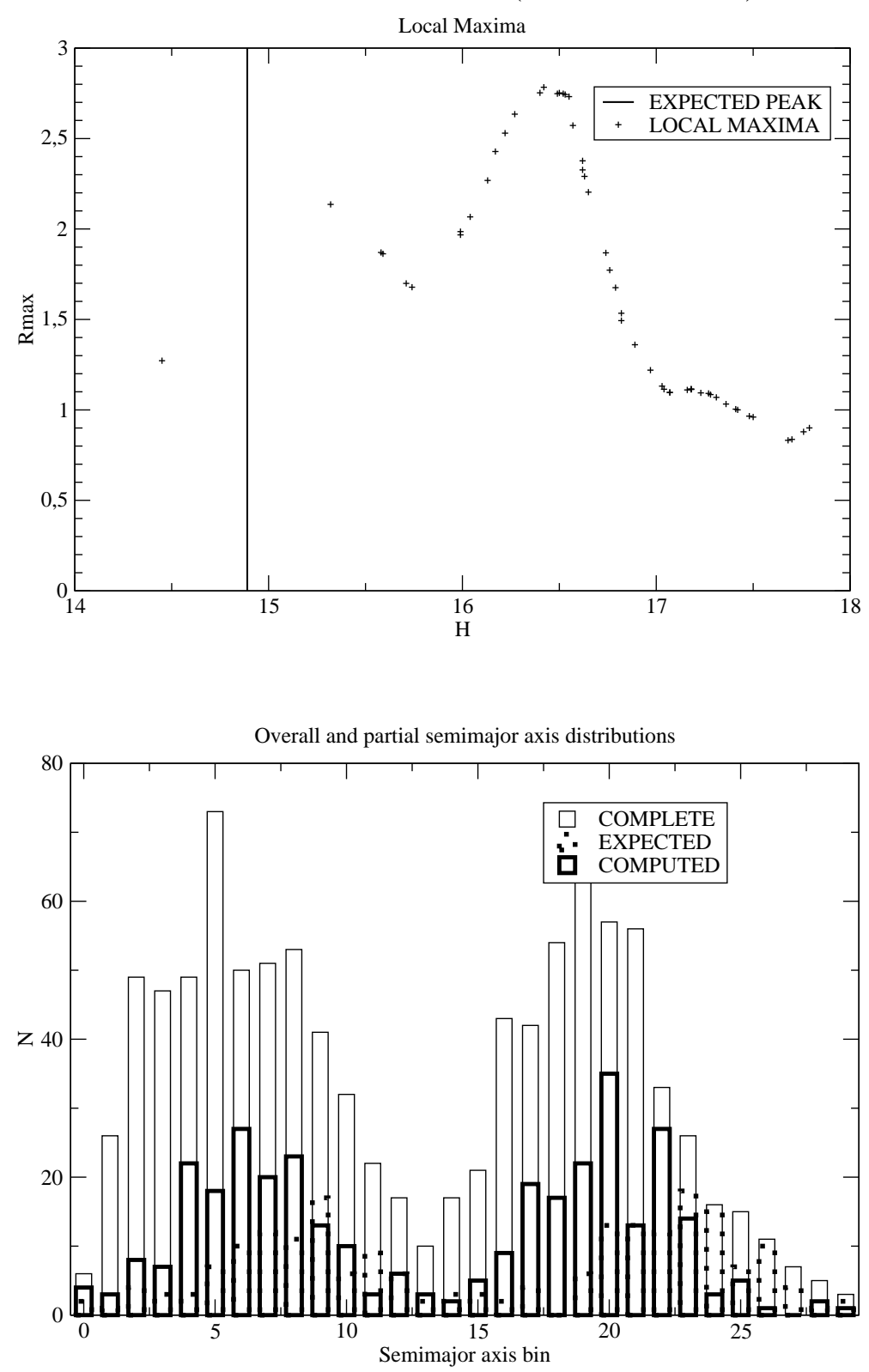

Figure 9: Top panel: The same as Figure 7, but for family 163. Note that we assumed as significant the first maximum on the right of the vertical line. Bottom panel: The distribution of semimajor axis in 30 bins, for the complete family, for the bodies around the computed maximum, and for the bodies around the expected maximum (again +/$1 \mathrm{mag})$. 
FAMILY 20 MASSALIA


Figure 10: Top panel: The same as Figure 7, but for family 20. Note that we assumed as significant the first maximum on the right of the vertical line Bottom panel: The H-a "V-plot of family 20.As it is possible to see, some footprints of depleted regions can be seen in the lowest part of the plot, where we have very few bodies. Depleted regions at higher $H$ seem to be due to resonant processes. 
we have chosen as representative of the YORP effect because being close to the maximum expected on the basis of the ages derived by Spoto et al. (2015), is rather low, and our assignation is less convincing. Note, however, that in the large majority of these cases (at least for 5 out of 7 ) the computed maximum corresponds to bright objects, and difficulties are not unexpected in these cases. Thus we decided to keep the values.

We have to note here that such a choice introduces a bias, when in the following we compare the maxima computed by our method with those expected on the basis of the ages (see Figure 16), obtaining a very good fit. By taking into account also these cases, the resulting fit is not entirely realistic. We are going to show, however, that the fit is satisfactory even when we limit our analysis only to the cases in which we have adopted the absolute maxima.

In the remaining cases the absolute maximum is not far from the predicted location, and our analysis is more successful. Note, however, that also in these cases we have two different situations: in some cases the maximum corresponds to bright objects, and the statistics is rather poor. In other cases, when the maximum involves higher magnitude bodies, the evidence is stronger.

As an example of the former case, we represent in Figures 11 and 12 the properties of the family 2076, for which the maximum is expected at about $H=12$, while the second case is represented by the Figure 13, corresponding to the family 845, and by the Figure 14 corresponding to the family 1128 .

As we have shown, more or less striking footprints of the YORP effect are present throughout our sample. In some cases we find a central depletion in a wide range of $H$. In these cases there is an immediate evidence of the effect. These families are for the most part corresponding (except for the obvious increase of the memberships) to those for which Vokrouhlický et al. (2006) evidenced the effect and estimated the ages, as reported also by Nesvorný et al. (2015). We can add to this class the family of (845) Naema. In some cases a significant depletion is limited to specific values of $H$, and a faint "eye" can be seen in the $\mathrm{V}$-plot. We can list here the families of (15) Eunomia, (31) Euphrosyne, (569) Misa, (606) Brangane. In other cases, sometimes also with a significant maximum, the depletion is not evident in the V-plot or is masked by other structural features (see Fig.11, bottom panel). Until now, we have used the ages estimated in Spoto et al. (2015) to obtain the YORPages, and then to predict the $H$ value for which we expect the maximum of $R$. We can also reverse the argument and the computations, using the 


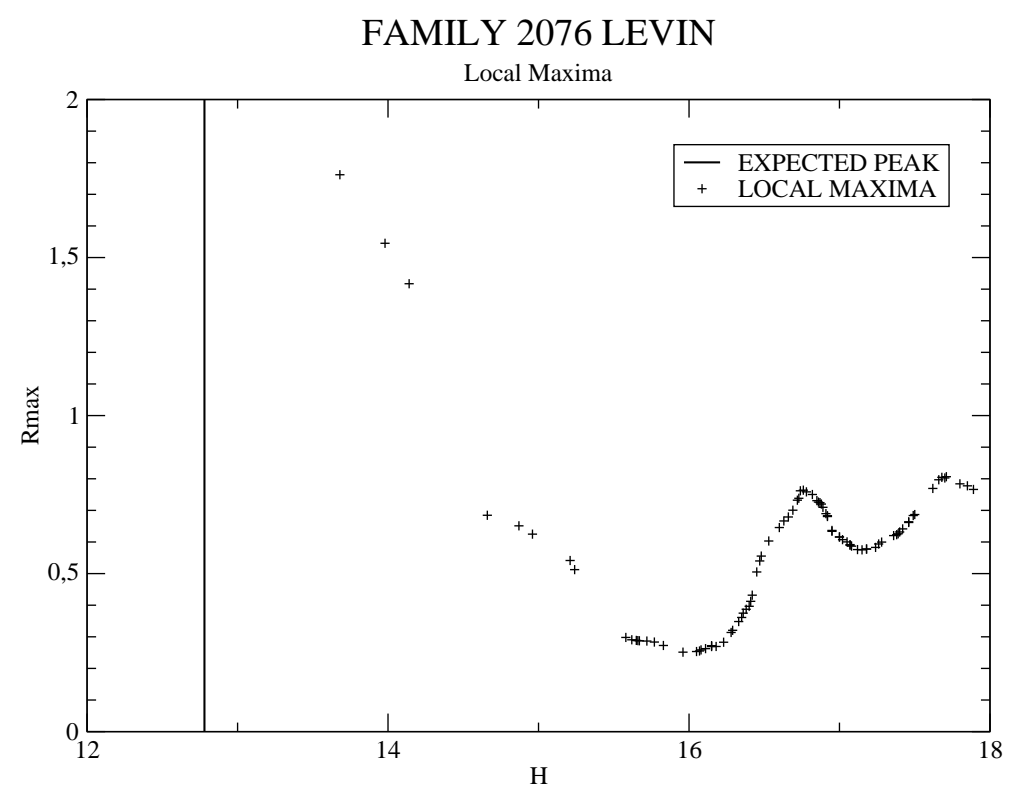

Figure 11: The same as Figure 7, but for the family 2076. Note that the absolute maximum is to the left, not far from the expected maximum.

computed maximum to estimate the age of the family, with a method which is completely independent on that used in Spoto et al. (2015). We will discuss this point more in detail in a forthcoming paper; however we remark that the comparison between the expected and the computed maximum corresponds to a comparison between the two age estimates; using Eqs. (6) and (7) it is easy to show that:

$$
H_{\text {comp }}-H_{\text {exp }}=2.5 \log _{10}\left(\tau_{\text {Yark }} / \tau_{Y O R P}\right)
$$

where the subscripts of $\tau$ refer to the effect used to estimate the age of the family under consideration.

We can thus move to a more synthetic approach, looking for the most relevant correlations. First, we analyze the possible correlation between the YORP-age and the height of the maximum $R$. The corresponding plot is represented in Figure 15. We plot in the figure only those families for which the value of $R$ is reported in Table 2. Here and below, the families with two estimated ages (and thus with two YORP-ages) are represented twice, but are correctly weighted in the computation of the regression coefficients. There is a small anti-correlation $R-Y O R P$ - age, probably not meaningful, 
FAMILY 2076 LEVIN

Overall and partial semimajor axis distribution
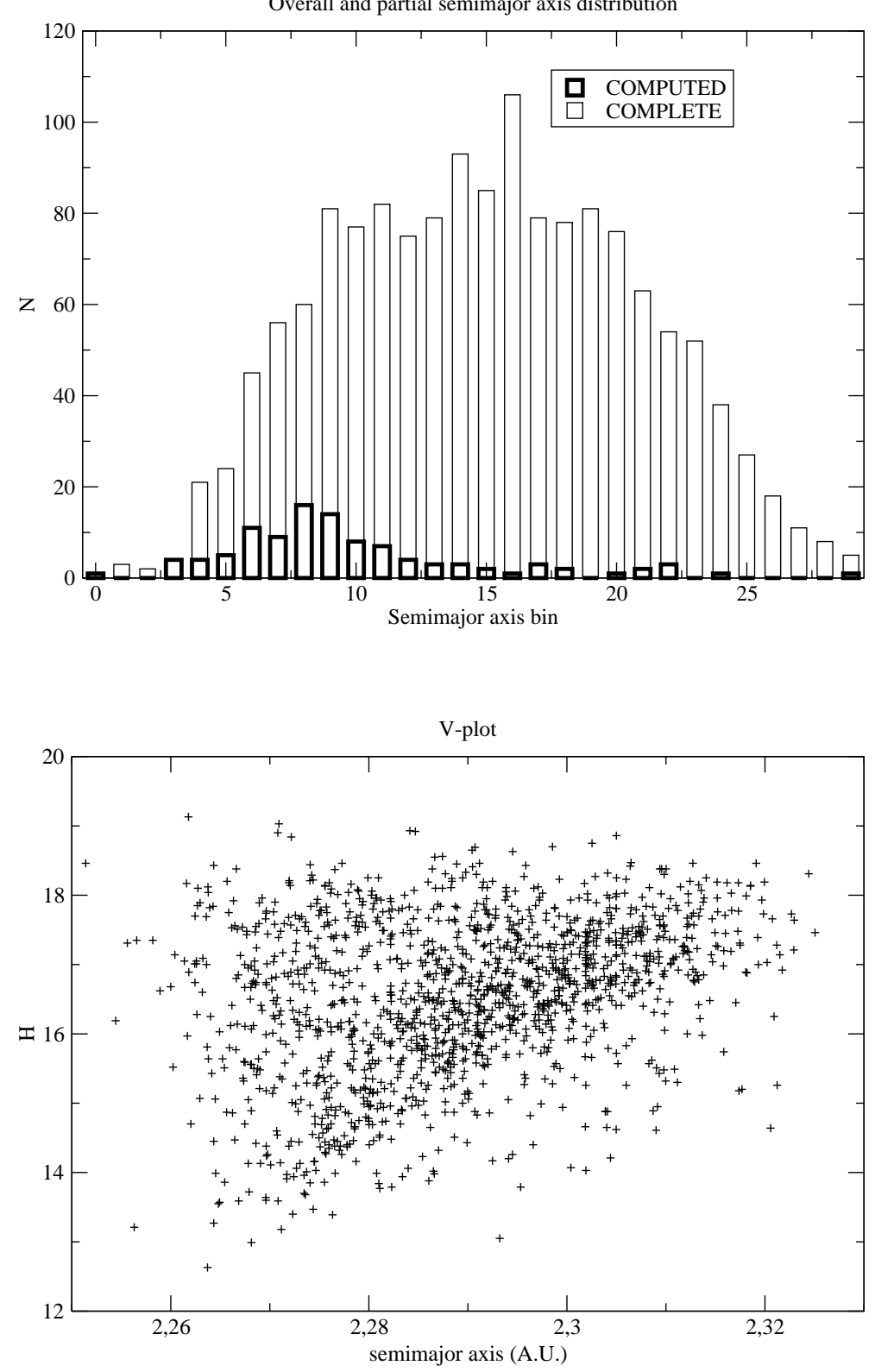

Figure 12: Top panel: The distribution of semimajor axis in 30 bins, for the complete family 2076, and for the (fewer) bodies around the computed maximum. Bottom panel: The V-plot. As can be expected, the depleted region, corresponding to few low $\mathrm{H}$ bodies, is not apparent in the plot. 


\section{FAMILY 845 NAEMA}
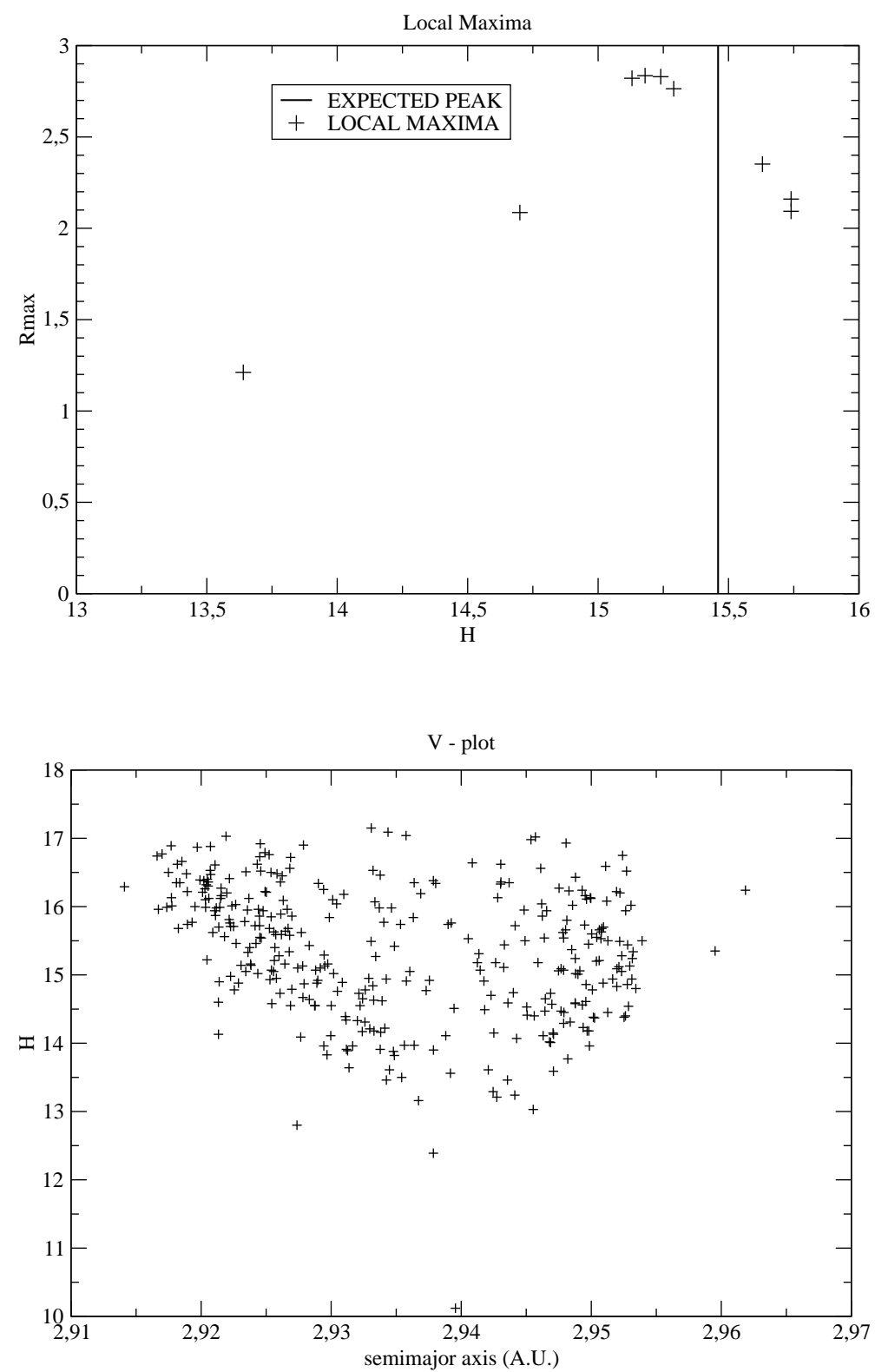

Figure 13: Top panel: the same as Figure 7, but for the family 845 . Note that there is an evident truncation (at $a \simeq 2.95$ ) of the family due to the $7: 3$ resonance. However the region of central depletion is located at a significantly smaller $a \simeq 2.94$, thus the reliability of our results is not strongly affected. Bottom panel: the a-H V plot of the family. 
FAMILY 1128 ASTRID
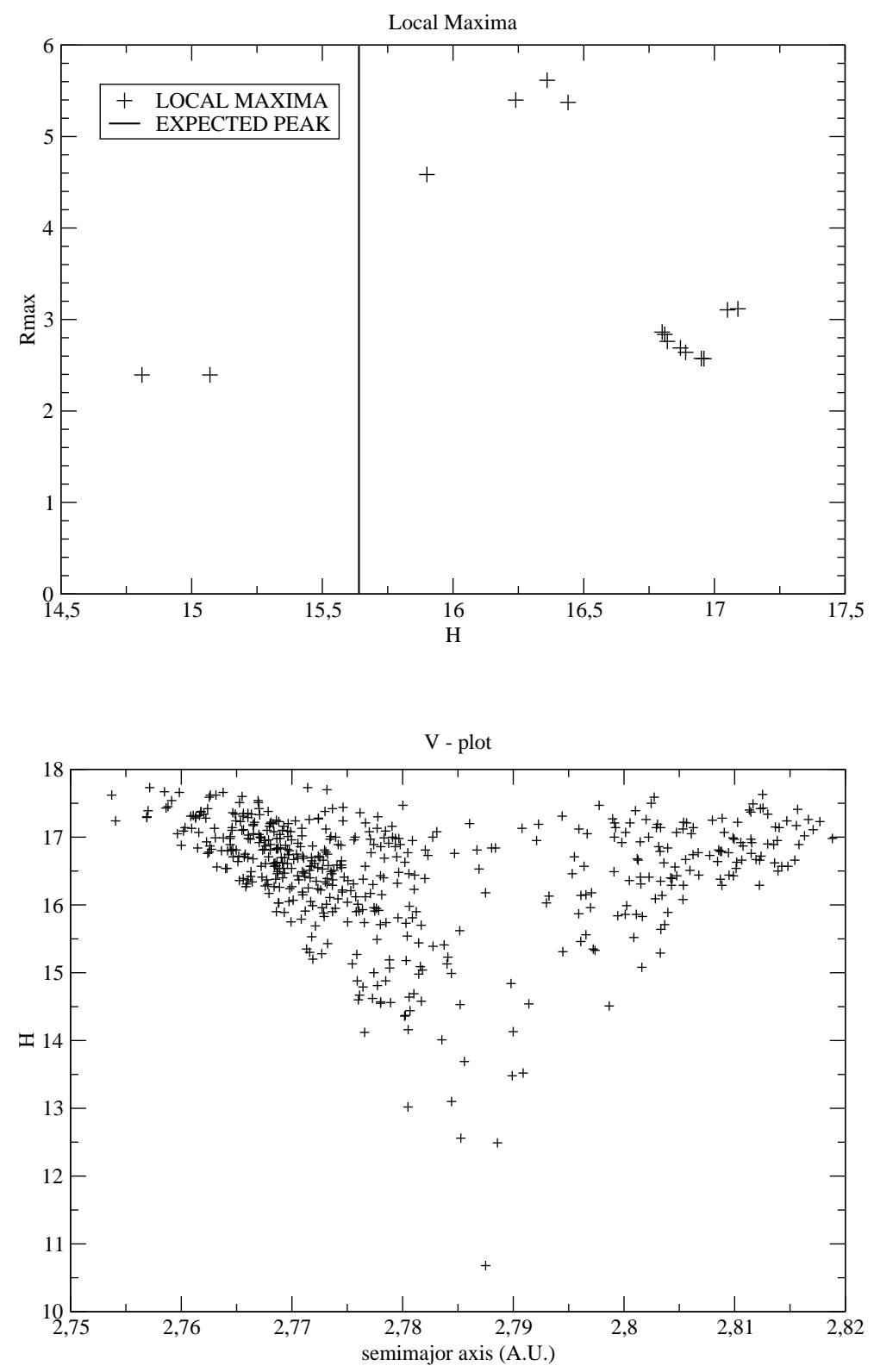

Figure 14: Top panel: the same as Figure 7, but for the family 1128. Bottom panel: the $\mathrm{a}-\mathrm{H} \mathrm{V}$ - plot of the family. 
VALUE OF THE MAXIMUM VS YORP-AGE

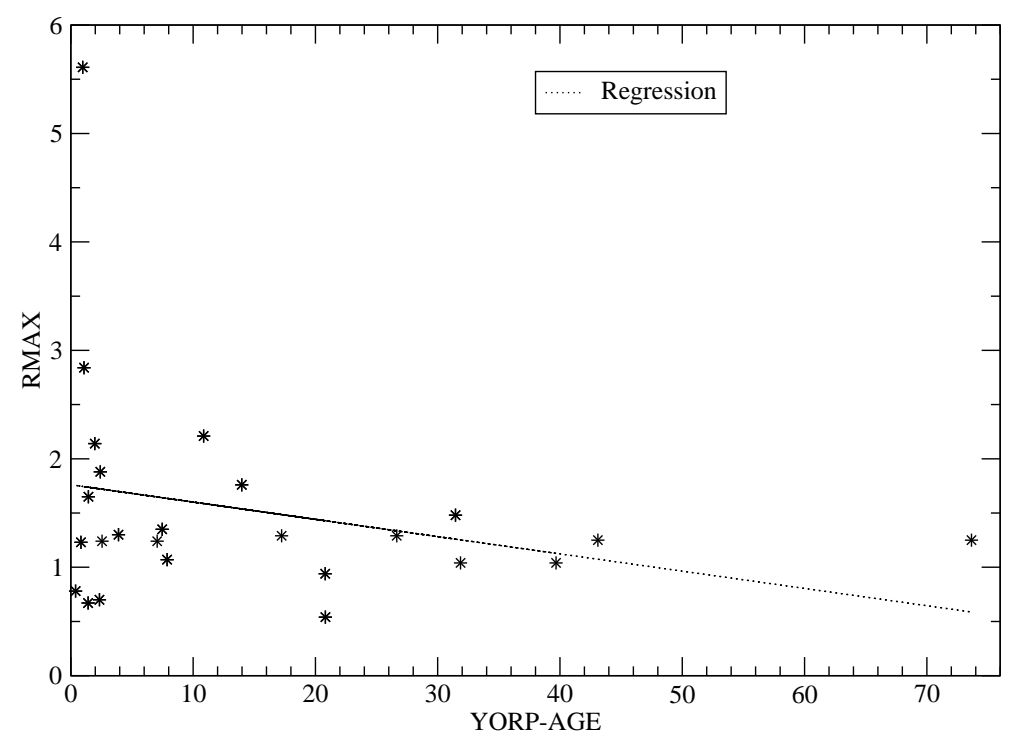

Figure 15: The maximum used value of $R$ as function of the YORP-age. We have included only the cases for which the value of $R$ is explicitely reported in Table 2 .

even if some further scrutiny might be worthwhile. Note that we have not included the cases where a secondary maximum was selected, but $R$ was small (see note (5) in Table 2 and discussion above). The inclusion of these data anyway does not alter significantly the outcome.

The most interesting outcome of our analysis is the correlation between the computed location of the maximum $R$ (as function of $H$ ) and that expected by the YORP-age estimated using the slope of the V-plot (Spoto et al., 2015), with the assumptions and simplifications discussed above and used to prepare the Table 1, and the other relevant parameters (semimajor axis, albedo). As explained above, this correlation corresponds to a correlation between the age estimates.

In Figure 16 we plot the computed vs. the expected location in $H$. We use different symbols for the 17 families for which we use the absolute maximum and for the other 11 for which a secondary maximum was adopted (at least four of these latter are, however, completely reliable, see discussion above). The figure shows a very strong correlation; the fitted line has a regression coefficient .84 for the "best" data, and .92 for all the data (in an ideal world the coefficient should be equal to one). 


\section{COMPUTED VS EXPECTED MAXIMA}

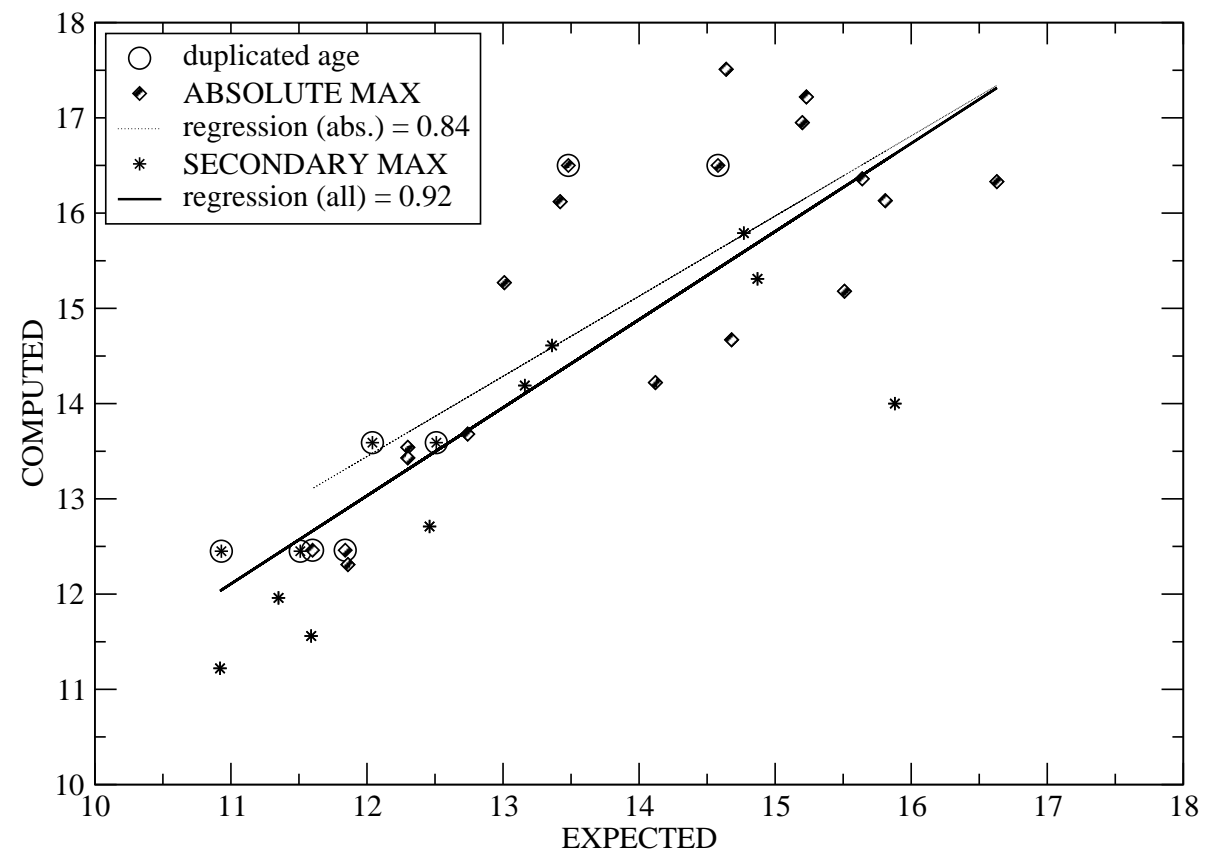

Figure 16: The $H$-location of the computed maximum vs. the value expected according to the age estimated by the slope of the $\mathrm{V}$-plot. The cases in which a secondary maximum has been adopted are represented with different symbols. The circles indicate families with two estimated ages. 
This correlation is also very significant. Note that an experiment performed with a different $f(A)$ (see Section 2), i.e. with $f(A)=A$ (corresponding to the limit of infinite thermal conductivity), gives a significantly worse correlation.

There is, however, a certain offset between the computed and expected values: they do not have the same average. This is not surprising because of the uncertainties in the calibration of Yarkovsky drift ages in Spoto et al. (2015), and, even more, because of the uncertainties concerning the YORP eye theory, discussed in detail in Sect. 3. We recall also that our Ansatz, by which the YORP eye (or something similar) should appear exactly at the $H$ value corresponding to bodies which have undergone one YORP cycle, is certainly reasonable but has to be considered rather qualitative. In order to obtain the same averages, we should add 0.912 to the expected values, i.e. we need to re-calibrate the family ages, and/or the constant in Eq. (7), and/or the Ansatz above. Doing this exercise causes essentially a translation along the abscissa in the Fig. 16. However, in a case (family of (1547) Nele) for which we have chosen a secondary maximum, after the recalibration the most natural choice is different. The three cases labelled as "OUT" remain critical also after the re-calibration, even if the expected peaks are shifted closer to the local maximum corresponding to the lowest $H$-value: these maxima correspond to very low values of $R$. The only change done did not, obviously, affect the regression coefficient of the "best" data, while the one corresponding to the complete sample rises up to the impressive value of .97.

\section{Conclusions}

We have introduced a method enabling a synthetic analysis of the observable consequences of the YORP effect in the subset of 31 families for which the age has been estimated (Spoto et al., 2015). The use of the central depletion parameter, explained in Section 4, allowed us to find significant YORP footprints in several families. In other cases traces of this effect can be identified, but the evidence is either weak or ambiguous. In a few cases (3 over 31, according to the results of Section 5) the theory predicts that it is not possible to find traces in the statistically significant range of absolute magnitude $H$. Even within the limitations discussed in Section 3, due to the extreme complexity of the physical processes affecting the observable data, and to the uncertainties of the parameters and of the theory, the results are interesting, and support the theoretical considerations. 
The location of the YORP-eye in the V-plot gives an indication of the age of the family. Note also that for some (old) families the YORP-eye corresponds to bright objects, which are less numerous (sometimes too few for a statistical analysis). This fact accounts mostly for the different success of our analysis in the individual cases.

Our analysis has been limited to the families for which an estimate of the age is available, being derived by means of the independent parameters (the slope of the borders of the V-plot). This estimate of the age provides a prediction on the location of the YORP-eye. A comparison between the locations computed by our method and those predicted on the basis of the family ages exhibits a good overall agreement (the best fit regression coefficient of 0.97 , instead of the "ideal" unity). This general result, on one side, supports the basic ideas of the present paper, including the simplifications concerning thermal conductivity and albedos; on the other hand, it strengthens also the reliability of the age estimates obtained by Spoto et al. (2015). In principle, the required re-calibration (see the discussion in Section 5) may furnish also an indication on the effectiveness of the YORP effect. The slowly growing set of observational data (Vokrouhlický et al., 2015) provides a few rather scattered estimates; note, however, that the intensity (and thus the timescale) of the YORP effect depends on the individual properties of the objects. Our results may give an indication on the average properties of the family asteroids, relevant for the effect.

The agreement between the two estimates of the age in the individual cases is not equally exciting. In other words, the scatter around the fit line in Figure 16 is not always small; while for some families the relative error between the ages computed in the two ways is rather low (a few tenths), for other cases we have an uncertainty by a factor two or even more. This fact may also provide some information about the "error bars" concerning the individual points in the plot. We are not able, at this stage, to compute reliable error bars. Some uncertainties may come out from the algorithm itself ("internal errors"); for instance the results may change with the size of the "running box" used in the computations. However, they may affect the individual cases, not the overall statistical results, which are the main result of the present paper. We have already discussed the several "physical" sources of error, which are expected to be rather large in the individual cases, and might also, in principle, entail some systematic error. However, a sensible analysis of the individual cases requires, in our opinion, a more thorough, "case-per-case" exploitation of all the available data and of the information 
on all possible sources of error, i.e. taking into account also those which are typical of every family, such as resonances, secondary events, and so on. We intend to improve our analysis in the future, with a more explicit comparison of the age estimates, taking into account also the increasing number of families with an estimated age (Milani et al., 2016).

Acknowledgments We are grateful to A. Cellino for useful suggestions that make the list of the sources of error more complete, and helped us to clarify some points. We are also grateful to A. Milani for stimulating discussions and suggestions and to A. La Spina for some comments. Useful comments and suggestions have been provided by the referees M.Dykhuis and D. Vokrouhlický. ZK acknowledges the support from the Ministry of Education, Science and Technological Development through project OI176011. PP acknowledges the funding by University of Pisa.

\section{References}

Bottke, W. F., Vokrouhlický, D., Rubincam, D. P., Brož, M.: Dynamical evolution of asteroids and meteoroids using the Yarkovsky effect. In Asteroids III, (Bottke, W.F. Jr., Cellino, A., Paolicchi, P., and Binzel, R.P., Eds.)), Univ. of Arizona Press, Tucson, pp. 395-408 (2002)

Bottke, W.F., Vokrouhlický, D., Rubincam, D.P., and Nesvorný, D. The Yarkovsky and Yorp Effects: Implications for Asteroid Dynamics, Ann. Rev. Earth Planet. Sci. 34, 157-191. (2006)

Bottke, W.F., Vokrouhlický, D., Walsh, K.J., Delbo, M., Michel, P., Lauretta, D. S., Campins, H., Connolly, H.C., Scheeres, D.J., Chelsey, S.R.: In search of the source of asteroid (101955) Bennu: Applications of the stochastic YORP model. Icarus 247, 191-217 (2015)

Breiter, S., and Vokrouhlický, D.: Yarkovsky-O'Keefe-Radzievskii-Paddack effect with anisotropic radiation, MNRAS 410, 2807-2816 (2011)

Capek, D. and Vokrouhlický, D.: The YORP effect with finite thermal conductivity, Icarus 172, 526-536 (2004)

Chesley, S. R., Ostro, S. J., Vokrouhlický, D., Čapek, D., Giorgini, J.D., Nolan, M.C., Margot, J.-L., Hine, A.A., Benner, L.A.M., Chamberlin, 
A.B.: Direct detection of the Yarkovsky effect via radar ranging to the near Earth asteroid 6489 Golevka, Science, 302, 1739-1742 (2003)

Cotto-Figueroa, D., Statler, T.S., Richardson, D.C., and Tanga, P.: Coupled Spin and Shape Evolution of Small Rubble-pile Asteroids: Self-limitation of the YORP Effect, Astrophys. J. 803, Issue 1, article id. 25 (2015)

Dell'Oro A. and Cellino A.: The random walk of Main Belt asteroids: orbital mobility by non-destructive collisions, MNRAS 380, 399-416 (2007)

Farinella, P., Vokrouhlický, D., and Hartmann, W. K.: Meteorite delivery via Yarkovsky orbital drift. Icarus, 132, 378-387 (1998)

Farinella P., and Vokrouhlický D.: Semimajor Axis Mobility of Asteroidal Fragments, Science, 283, 1507-1510 (1999)

Hanuš, J., Ďurech, J., Brož, M., Warner, B. D., Pilcher, F., Stephens, R., Oey, J., Bernasconi, L., Casulli, S., Behrend, R., Polishook, D., Henych, T., Lehký, M., Yoshida, F., and Ito, T.: A study of asteroid pole-latitude distribution based on an extended set of shape models derived by the lightcurve inversion method. Astron. Astrophys. 530, id.A134 (2011)

Kryszczyńska, A., La Spina, A., Paolicchi, P., Harris, A.W., Breiter, S., and Pravec, P.: New findings on asteroid spin-vector distributions. Icarus 192, 223-237 (2007)

La Spina, A., Paolicchi, P., Kryszczyńska, A., and Pravec, P.: Retrograde spins of near-Earth asteroids from the Yarkovsky effect. Nature 428, 400401 (2004)

La Spina, A., Paolicchi, P., and Penco, U.: Yarkovsky-evolved asteroid dynamical families: a correlation between their present properties and the impact geometry? American Astronomical Society, DPS meeting 37, 15.14; Bull. American Astron. Soc. 37, 641 (2005)

Micheli, M., and Paolicchi, P.: YORP effect on real objects. I. Statistical properties. Astron. Astrophys. 490, 387-391 (2008)

Milani, A., Cellino, A., Knežević, Z., Novaković, B., Spoto, F., and Paolicchi, P.: Asteroid families classification: Exploiting very large datasets. Icarus 239, 46-73 (2014) 
Milani, A., Spoto, F., Knežević, Z., Novaković, B, and Tsirvoulis, G.: Families classification including multiopposition asteroids. In: Asteroids: New Observations, New Models. Proceedings IAU Symposium 318 (Chesley, S., Farnocchia, D., Jedicke, R., and Morbidelli, A., Eds.), pp. 1-18, Cambridge University Press, Cambridge, in press. (2016)

Muinonen, K., Belskaya, I.N., Cellino, A., Delbò, M., Levasseur-Regourd, A.-C., Penttilä, A., Tedesco, E.F.: A three-parameter magnitude phase function for asteroids. Icarus 209, 542-555 (2010)

Nesvorný, D., and Vokrouhlický, D.: Analytic theory for the YORP effect on obliquity. Astron. J., 136, 291-299 (2008)

Nesvorný, D., Brož, M., and Carruba,V.: Identification and Dynamical Properties of Asteroid Families. In Asteroids IV (Michel, P., DeMeo, F.E, and Bottke, W.F., Eds.), University of Arizona, Tucson , pp. 297-322 (2015)

Paolicchi P., and Kryszczyńska, A.: Spin vectors of asteroids: Updated statistical properties and open problems. Planet. Space Sci. 73, 70-74 (2012)

Pravec, P., Harris, A.W., and Michalowski, T.: Asteroid Rotations. In: Asteroids III (Bottke, W.F. Jr., Cellino, A., Paolicchi, P., and Binzel, R.P., Eds.), Arizona Univ. Press, Tucson, pp. 113-122 (2002)

Pravec, P., Harris, A.W., Kušnirák, P., Galád, A., and Hornoch, K.: Absolute magnitudes of asteroids and a revision of asteroid albedo estimates from WISE thermal observations. Icarus 221, 365-387 (2012)

Rubincam D.P.: Radiative Spin-up and Spin-down of Small Asteroids, Icarus 148, 2-11 (2000)

Slivan, S.M.: Spin vector alignment of Koronis family asteroids. Nature 419, 49-51 (2002)

Spoto, F., Milani, A., and Knežević, Z.: Asteroid family ages. Icarus 257, 275-289 (2015)

Statler, T.S.: Extreme sensitivity of the YORP effect to small-scale topography, Icarus 202, 502-513 (2009) 
Vereš, P., Jedicke, R., Fitzsimmons, A., Denneau, L., Granvik, M., Bolin, B., Chastel, S., Wainscoat, R.J., Burgett, W.S., Chambers, K.C., Flewelling, H., Kaiser, N., Magnier, E.A., Morgan, J.S., Price, P.A., Tonry, J.L., and Waters, C.: Absolute magnitudes and slope parameters for 250,000 asteroids observed by Pan-STARRS PS1 - Preliminary results. Icarus 261, 34-47, (2015)

Vokrouhlický, D., and Čapek, D.,YORP-Induced Long-Term Evolution of the Spin State of Small Asteroids and Meteoroids: Rubincam's Approximation. Icarus 159, 449-467, (2002)

Vokrouhlický, D., Nesvorný, D., and Bottke W. F.: The vector alignments of asteroid spins by thermal torques. Nature,425, 147-151 (2003)

Vokrouhlický, D., Brož, M., Bottke, W.F., Nesvorný, D., Morbidelli, A.: Yarkovsky/YORP chronology of asteroid families. Icarus 182, 118-142 (2006)

Vokrouhlický, D., Bottke, W. F., Chesley S.R., Scheeres D.J. and Statler T.S.: The Yarkovsky and YORP effects. In Asteroids IV (Michel, P., DeMeo, F.E, and Bottke, W.F., Eds.), University of Arizona, Tucson, pp. 509-531 (2015) 


\begin{tabular}{|l|l|l|l|l|l|l|l|l|}
\hline COREFAM & MEMB & AMIN & AMAX & AM & ALB. & AGE & YAGE & \\
\hline 3 & 1532 & 2.621 & 2.700 & 2.660 & 0.25 & $550 / 370$ & $19.43 / 13.07$ & $(1)$ \\
4 & 10065 & 2.256 & 2.482 & 2.369 & 0.35 & $930 / 1906$ & $58 . / 118.9$ & $(1)$ \\
10 & 2989 & 3.067 & 3.241 & 3.154 & 0.07 & 1349 & 9.49 & \\
15 & 9117 & 2.521 & 2.733 & 2.627 & 0.26 & $1955 / 1144$ & $73.65 / 45.1$ & $(1)$ \\
20 & 7525 & 2.334 & 2.474 & 2.404 & 0.25 & 182 & 7.87 & \\
24 & 5425 & 3.062 & 3.242 & 3.152 & 0.07 & $2447 / 3782$ & $17.24 / 26.64$ & $(1)$ \\
31 & $1714^{*}$ & 3.075 & 3.225 & 3.150 & 0.06 & 1235 & 7.47 & \\
158 & 7071 & 2.816 & 2.985 & 2.900 & 0.24 & 1750 & 49.94 & \\
$163 / 5026$ & 998 & 2.331 & 2.418 & 2.374 & 0.05 & 221 & 1.96 & \\
170 & 2762 & 2.523 & 2.673 & 2.598 & 0.26 & 1932 & 74.42 & $(2)$ \\
375 & 784 & 3.096 & 3.241 & 3.168 & 0.06 & 3483 & 20.82 & $(2)$ \\
396 & 477 & 2.728 & 2.752 & 2.740 & 0.11 & 96 & 1.40 & \\
434 & 1663 & 1.883 & 1.988 & 1.940 & 0.38 & 206 & 20.8 & \\
480 & 1345 & 2.538 & 2.732 & 2.635 & 0.29 & $763 / 950$ & $31.87 / 39.68$ & $(1)$ \\
569 & 621 & 2.623 & 2.694 & 2.658 & 0.06 & 284 & 2.41 & \\
606 & 282 & 2.571 & 2.597 & 2.584 & 0.12 & 46 & 0.83 & \\
668 & 1634 & 2.744 & 2.811 & 2.777 & 0.06 & 501 & 3.89 & \\
808 & 1197 & 2.705 & 2.810 & 2.757 & 0.25 & 330 & 10.85 & \\
845 & 344 & 2.914 & 2.962 & 2.938 & 0.06 & 156 & 1.08 & \\
847 & 3059 & 2.713 & 2.819 & 2.766 & 0.24 & 1003 & 31.46 & $(3)$ \\
1128 & 515 & 2.754 & 2.819 & 2.786 & 0.05 & 150 & 0.97 & \\
$93(1272)$ & 2329 & 2.720 & 2.816 & 2.768 & 0.28 & 1103 & 40.31 & $(2)$ \\
$293(1521)$ & 778 & 2.832 & 2.873 & 2.852 & 0.17 & $338 / 122$ & $7.06 / 2.55$ & $(1)$ \\
1547 & 290 & 2.638 & 2.650 & 2.644 & 0.36 & 15 & 0.77 & \\
1658 & 751 & 2.544 & 2.626 & 2.585 & 0.26 & 464 & 18.05 & $(2)$ \\
1726 & 1927 & 2.754 & 2.819 & 2.786 & 0.05 & 332 & 2.14 & \\
2076 & 1484 & 2.251 & 2.325 & 2.288 & 0.20 & 366 & 13.98 & \\
3330 & $1113 *$ & 3.123 & 3.178 & 3.150 & 0.05 & 464 & 2.34 & \\
3815 & 538 & 2.561 & 2.585 & 2.567 & 0.05 & 51 & 0.39 & \\
3827 & 985 & 2.705 & 2.768 & 2.736 & 0.07 & 154 & 1.44 & \\
10955 & 669 & 2.671 & 2.739 & 2.705 & 0.25 & 470 & 16.06 & \\
\hline
\end{tabular}

Table 1: Summary of the data for the families used in the computations. For every family (Corefam= number of the core family - see (Spoto et al., 2015)) we list the number of members (MEMB). Note that the stars refer to two cases in which we have dropped one member of the family with no $H$-value listed in our database. We list also the minimum (AMIN), maximum (AMAX) and average value of the semimajor axis (AM), the geometrical albedo (ALB), the age (AGE) in $M y$ and the computed YORPAGE (see text). In the notes (1) indicates duplicated families; (2) refers to "onesided" families and (3) to a case for which only one age has been taken as meaningful. See text for details. Note also that the family $163 / 5026$ is the 36 mbination of the nominal family 163 with the family 5026, and we list as families 293 and 93 those for which in reality the largest members (in parentheses) are the asteroids 1521 and 1272 (Spoto et al., 2015). 


\begin{tabular}{|c|c|c|c|c|c|c|}
\hline FAM & YAGE & EXP & COMP & RMAX & NOTES & CALIB \\
\hline 3 & $19.43 / 13.07$ & $12.38 / 12.81$ & OUT & & $(1)$ & $13.29 / 13.72$ \\
\hline 4 & $58 . / 118.9$ & $11.19 / 10.41$ & OUT & & (1) & $12.10 / 11.32$ \\
\hline 10 & 9.49 & 13.16 & 14.19 & & $(5)$ & 14.07 \\
\hline 15 & $73.65 / 43.1$ & $10.93 / 11.51$ & 12.45 & 1.25 & $(1,3)$ & $11.84 / 12.43$ \\
\hline 20 & 7.87 & 13.36 & 14.61 & 1.07 & (4) & 14.27 \\
\hline 24 & $17.24 / 26.65$ & $12.51 / 12.04$ & 13.59 & 1.29 & $(1,3)$ & $13.42 / 12.95$ \\
\hline 31 & 7.47 & 13.42 & 16.12 & 1.35 & & 14.33 \\
\hline 158 & 49.94 & 11.35 & 11.96 & & $(5)$ & 12.27 \\
\hline $163 / 5026$ & 1.96 & 14.87 & 15.31 & 2.14 & $(2)$ & 15.78 \\
\hline 170 & 74.42 & 10.92 & 11.22 & & $(5)$ & 11.83 \\
\hline 375 & 20.82 & 12.30 & 13.54 & 0.54 & & 13.22 \\
\hline 396 & 1.41 & 15.23 & 17.22 & 0.67 & & 16.14 \\
\hline 434 & 20.8 & 12.30 & 13.43 & 0.94 & & 13.22 \\
\hline 480 & $31.87 / 39.68$ & $11.84 / 11.6$ & 12.46 & 1.04 & (1) & $12.75 / 12.52$ \\
\hline 569 & 2.41 & 14.64 & 17.51 & 1.88 & & 15.56 \\
\hline 606 & 0.83 & 15.81 & 16.13 & 1.23 & & 16.72 \\
\hline 668 & 3.9 & 14.12 & 14.22 & 1.3 & & 15.04 \\
\hline 808 & 10.86 & 13.01 & 15.27 & 2.21 & & 13.92 \\
\hline 845 & 1.08 & 15.51 & 15.18 & 2.84 & & 16.42 \\
\hline 847 & 31.46 & 11.86 & 12.31 & 1.48 & & 12.77 \\
\hline 1128 & 0.97 & 15.64 & 16.36 & 5.61 & & 16.55 \\
\hline $93(1272)$ & 40.31 & 11.59 & 11.56 & & (5) & 12.5 \\
\hline $293(1521)$ & $7.06 / 2.55$ & $13.48 / 14.58$ & 16.5 & 1.24 & (1) & $14.39 / 15.5$ \\
\hline 1547 & 0.77 & 15.88 & 14.97 & & $(5,6)$ & 16.79 \\
\hline 1547 & & & 16.38 & & (5) & 16.79 \\
\hline 1658 & 18.05 & 12.46 & 12.71 & & $(5)$ & 13.37 \\
\hline 1726 & 2.14 & 14.77 & 15.79 & & $(5)$ & 15.69 \\
\hline 2076 & 13.98 & 12.74 & 13.68 & 1.76 & & 13.65 \\
\hline 3330 & 2.34 & 14.68 & 14.67 & 0.7 & & 15.59 \\
\hline 3815 & 0.39 & 16.63 & 16.33 & 0.78 & & 17.54 \\
\hline 3827 & 1.44 & 15.20 & 16.95 & 1.65 & & 16.12 \\
\hline 10955 & 16.06 & 12.59 & OUT & & & 13.5 \\
\hline
\end{tabular}

Table 2: The Table summarizes the results of our computations. For every family (FAM= number) we repeat the YAGE reported in the Table 1 and the $H$-value of the expected maximum of $R$ (EXP). We list also the $H$-value corresponding to the maximum value of $\mathrm{R}$ resulting from our computations (COMP), and the corresponding $\mathrm{R}$ value of this maximum (RMAX). In some cases we write "OUT" in the corresponding column, meaning that the expected maximum is completely out of the explored range. We report also the $H$-value of the expected peak after the re-calibration (CALIB). The notes: (1): a duplicated family; (2) we have taken a secondary maximum, but with a high value of R; (3) we have taken a marginally secondary maximum, (4) we have taken a secondary maximum on the border, closest to the expected value; (5) we have taken a secondary maximum, and the $\mathrm{R}$ value is low; (6) in this case the recalibration implied a different choice of the secondary maximum (reported in the row below). See text for more details. 